\title{
Dynamical local field, compressibility and frequency sum rules for quasiparticles
}

\author{
Klaus Morawetz \\ Max-Planck-Institute for the Physics of Complex Systems, Noethnitzer Str. 38, 01187 Dresden, Germany
}

\begin{abstract}
The finite temperature dynamical response function including the dynamical local field is derived within a quasiparticle picture for interacting one-, two- and three dimensional Fermi systems. The correlations are assumed to be given by a density dependent effective mass, quasiparticle energy shift and relaxation time. The latter one describes disorder or collisional effects. This parameterization of correlations includes local density functionals as a special case and is therefore applicable for density functional theories. With a single static local field, the third order frequency sum rule can be fulfilled simultaneously with the compressibility sum rule by relating the effective mass and quasiparticle energy shift to the structure function or pair correlation function. Consequently, solely local density functionals without taking into account effective masses cannot fulfill both sum rules simultaneously with a static local field. The comparison to the Monte-Carlo data seems to support such quasiparticle picture.
\end{abstract}

05.30.Fk,21.60.Ev, 24.30.Cz, 24.60.Ky

\section{INTRODUCTION}

The response of an interacting Fermi system with the potential $V_{q}$ to an external perturbation is the basic source of our knowledge about the interaction and dynamical as well as statical properties of the system. This response function has been therefore a central issue of many body theories.

The density response function gives the variation of the density in terms of the external potential

$$
\delta n(q, \omega)=\chi(q, \omega) V^{\mathrm{ext}}(q, \omega) .
$$

The polarization is defined as the density variation in terms of the induced potential

$$
\delta n(q, \omega)=\Pi(q, \omega) \delta V^{\text {ind }}(q, \omega)
$$

where we suppress the notation of obvious $q$-dependence in the following. The induced potential itself is the sum of the external potential and the effective interaction potential $\left(V_{q}+f_{q}(\omega)\right) \delta n$

$$
\delta V^{\text {ind }}(\omega)=\left(V_{q}+f_{q}(\omega)\right) \delta n(\omega)+V^{\operatorname{ext}}(\omega) .
$$

Therefore, from (11)-(3) we have the relation between response and polarization

$$
\chi(\omega)=\frac{\Pi(\omega)}{1-\left(V_{q}+f_{q}(\omega)\right) \Pi(\omega)} .
$$

The local field $f_{q}(\omega)$ describes the modification in the restoring force brought about by particle correlations. This field prevents the particles from sampling the full effect of interaction at short distancest

The dielectric function relates now the induced densities to the external potential via

$$
\frac{1}{\epsilon(\omega)}=1+\frac{V_{q} \delta n(\omega)}{V^{\operatorname{ext}}(\omega)} \equiv 1+V_{q} \chi(\omega)
$$

such that the dielectric function reads

$$
\epsilon(\omega)=1-\frac{V_{q} \Pi(\omega)}{1-f_{q}(\omega) \Pi(\omega)} .
$$

The theoretical effort consists in determining the local field $f_{q}(\omega)$ which represents the local correlation and which depleted the induced potential by $f_{q}(\omega)=$ $-G(\omega) V_{q}$. As long as this local field is a dynamical one this is an exact relation. The different theoretical treatments differ in this local field corrections, for an overview seet. Mostly static approximations, $f_{q} \equiv f_{q}(0)=-V_{q} G$, have been proposed in the past. It has started with the pioneering work of Hubbard $\mathrm{B}$ who first introduced the notation of local field and took into account the exchangehole correction resulting in

$$
G_{H}=\frac{1}{2} \frac{q^{2}}{q^{2}+k_{f}^{2}}=\left\{\begin{array}{l}
\frac{1}{2} \frac{q^{2}}{k_{f}^{2}}+o\left(q^{3}\right) \\
\frac{1}{2}+o\left(1 / q^{2}\right)
\end{array} .\right.
$$

While this expression has established a remarkable improvement of the dielectric function in random phase approximation (RPA), it has been soon recognized insufficient due to the lack of self-consistency which leads the pair correlation function still to unphysical negative values. This has been repaired by Singwi et. al. 1 by using exchange-correlations

$$
\begin{aligned}
G_{S T L S} & =-\frac{1}{n} \int \frac{d k}{(2 \pi)^{3}} \frac{(k \cdot q)}{k^{2}}\left(S_{k-q}-1\right) \\
& =\left\{\begin{array}{c}
\gamma \frac{q^{2}}{k_{f}^{2}}+o\left(q^{3}\right) \\
1-g_{0}+o\left(1 / q^{2}\right)
\end{array}\right.
\end{aligned}
$$

with

$$
\gamma=-\frac{1}{s k_{f}} \int_{0}^{\infty} d q\left(S_{q}-1\right)
$$


where $s$ is the spin degeneracy and where the static structure factor

$$
\begin{aligned}
S_{q} & =-\int \frac{d \omega}{n \pi V_{q}} \frac{\operatorname{Im} \epsilon^{-1}(\omega)}{1-\mathrm{e}^{-\beta \omega}} \\
& =\int \frac{d \omega}{n \pi} \frac{1}{1-\mathrm{e}^{-\beta \omega}} \operatorname{Im} \frac{\Pi(\omega)}{1-\left(f_{q}(\omega)+V_{q}\right) \Pi(\omega)}
\end{aligned}
$$

with inverse temperature $\beta=1 / T$ is linked to the pair correlation function via

$$
g_{r}-1=\frac{1}{n} \int \frac{d q}{(2 \pi)^{3}} \mathrm{e}^{i q r}\left(S_{q}-1\right) .
$$

This provides a self-consistent problem in solving dielectric function, structure function and static local field simultaneously. The advantages of this result compared to the Hubbard result with respect to the pair correlation function and large wavevector limit has been discussed by . Recent comparisons with molecular dynamics simulations for a hard sphere gas is presented in 6 where a good agreement is found for thermodynamical properties.

The expression (8) has been improved further by Pathak and Vashishtat demanding that the responsf function should fulfill the third order frequency sum rules which resulted into

$$
\begin{aligned}
G_{P V} & =-\frac{1}{n} \int \frac{d k}{(2 \pi)^{3}} \frac{(k \cdot q)^{2}}{q^{4}} \frac{V_{k}}{V_{q}}\left(S_{k-q}-S_{k}\right) \\
& =\left\{\begin{array}{c}
\frac{2}{5} \gamma \frac{q^{2}}{k_{f}^{2}}+o\left(q^{3}\right) \\
\frac{2}{3}\left(1-g_{0}\right)+o\left(1 / q^{2}\right)
\end{array}\right.
\end{aligned}
$$

leading to the improved small distance limit discussed in 9 . The difference at short distance to $G_{S T L S}$ comes from the motion of particles inside the correlation hole which is condensed in the dynamical behavion 10 .

If one takes into account the difference between uncorrelated and correlated kinetic energy 11 . 1 one obtains an additional $-\Delta E=-\frac{2}{n^{2} V_{q}}\left(E_{i n t}-E\right)$ term to $G_{P V}$. This comes from the difference in correlated and uncorrelated occupation numbers which can be expressed by a coupling constant integration and can be linked via the virial theorem to density derivatives of the pair correlation function 1213.

Parallel to the above discussions there has been different imprpiements to derive local fields from the virial formulal 11.14 which have resulted into expressions known from density variations

$$
G_{V S}=\left(1+a n \frac{\partial}{\partial n}\right) G_{S T L S}
$$

with various $1 / 2 \leq a \leq 1$, see 14 . This procedure satisfy the compressibility sum rule almost exactly.

In 11 it was shown for Coulomb systems that one cannot construct a static local field factor which fulfills both limits, the compressibility and the third order sum rule (32) since it would violate the theorem of Ferrell, $d^{2} E_{0} / d\left(e^{2}\right)^{2} \leq 0$. The same conclusion are obtained in 15 using the virial theorem.

Therefore the concentration is now mostly focussed on the sonstruction of dynamical local field correction 13,1618 . The quantum versions of the SingwiTosi-Land-Sjölander, $G_{S T L S}$, and_/ahishta-Singwi, $G_{V S}$, theories have been discussed in 16.1920 . These lead to positive values for the pair distribution function at short distances valid for rather low densities 6 . There it has been focussed on dynamical properties of the dynamic local field. While the high frequency limit is monotonic and similar to STLS and VS, the static limit can even exhibit peaked structures which can give rise to charge density waves underlying the nontrivial character of dynamical behavior. Unfortunately even the dynamic quantum version of the Singwi-Tosi-Land-Sjölapder local field cannot fulfill the compressibility sum rule 21 . We will show here that from a dynamical local field one can derive a static local field fulfilling both sum rules simultaneously if one takes into account the effective mass. This will resolve the puzzle of sum rules.

Recent improvements of the response function re $^{\mathrm{b}}$ basically due to numerical studies of Monte Carlo22 25 or molecular dynamical simulations 26.6. An interesting first principle numerical scheme is to solve the time dependent Kadanoff and Baym equations including an external field27. Due to the variation of internal lines, already a Born diagram leads to a linear response which includes high order vortex corrections fulfilling sum rules consistently. The third order frequency sum rule regains importance for reduced dimensional layered structures2, 28.29 . All results in this paper here can be straight forwardly generalized also to reduced dimensions as given in appendix B for one, two and three dimensions. This could have an impacton recent discussions of two-layered electron gasses 30 32.

Here we want to return to the analytical investigations and will show that there exist a possibility to fulfill with one static local field correction both requirements, the third order sum rule and the compressibility sum rule. This is performed by working within a quasiparticle picture determining the effective mass appropriately. Within the frame of the quasiparticle picture we will derive an explicit expression for the dynamical local field factor which leads to the desired static limits. We obtain the identity

$$
\begin{aligned}
G & =G_{P V}+\frac{2}{n^{2} V_{q}}\left(E-E_{\text {int }}\right) \\
& \equiv-\frac{1}{V_{q}}\left(\frac{\partial \Delta}{\partial n}-\frac{1}{2 n m} \frac{\partial \ln m}{\partial \ln n}\left(\frac{\Pi_{2}(0)}{\Pi_{0}(0)}-\frac{q^{2}}{4}\right)\right)
\end{aligned}
$$

with moments of the polarization given in appendix B and the effective mass $m(n)$ and selfenergy $\Delta(n)$. It will provide a recipe how to construct a quasiparticle picture by the knowledge of the structure factor at small distances from experiments or simulations2622, 6 . This in turn leads to an easy microscopic parameterization in 
terms of the effective mass and quasiparticle energy shift which could be compared directly to microscopic theories.

The underlying principle is analogous to the one found in literature 33.34 where a response function was parameterized explicitly fulfilling sum rules and different constraints. Other parameterizations can be found in 13 from variational approaches which are exact in the high density limit. A different line of constructing the response function uses the frequency moments resulting in recurrence relation 35 . 36 .

Here in this paper we will give an alternative approach which uses general parameterizations of the selfenergy in terms of a functional which might depend on the density, energy and current. We restrict here to a one component system though the generalization to multicomponent systems is straight forward 3738 and considered in different approache 39411 .

In the next chapter we review shortly the compressibility sum rule and the third order frequency sum rule. In chapter III we give the dynamical response for quasiparticles which-is a special case of the general structure derived earlier 2 . We show that the correct compressibility appears and the third order sum rule can be satisfied if the effective mass and quasiparticle energy is chosen appropriately. Chapter IV will present some numerical results on the unpolarized electron gas at zero temperature and the comparison with Monte Carlo simulations are discussed. Chapter V summarizes the results and in appendix $\mathrm{B}$ we give frequently occurring correlation functions and practical forms for calculation in one-, twoand three dimensions. While all formulas in the main text are written as three dimensional, they hold for oneand two dimensions as well. Only the explicit correlation functions in appendix $\mathrm{B}$ have to be used as outlined there. Also the often required long wavelength expansion of these correlation functions are given in appendix B 1. Appendix C finally is devoted to the short sketch of perturbation theory and the derivation of the used sum rules for one, two and three dimensions.

\section{DETERMINATION OF STATIC LOCAL FIELD FACTOR}

Let us discuss two different boundaries for the static limit of the local field. This will be the compressibility and the third order frequency sum rule.

\section{A. Compressibility}

First we have to know how the compressibility should look like. This is particularly simple in the quasiparticle picture which we will use. In the quasiparticle picture the one-particle distribution function is a Fermi distribution

$$
F(p)=\left(\mathrm{e}^{\beta\left(\frac{p^{2}}{2 m}+\Delta-\mu\right)}+1\right)^{-1}
$$

where the density dependent effective mass $m$ and the selfenergy shift $\Delta$ are obtained either from microscopic calculations or, as proposed here, from the sum rules. Thorough the paper we will understand now the masses as effective masses.

From microscopic approaches the effective selfenergy shift and the effective mass are coming from the knowledge of the selfenergy $\sigma(p, \omega)$ which determines the quasiparticle energy $\epsilon$ via

$$
\epsilon=\frac{p^{2}}{2 m_{0}}+\sigma(p, \epsilon) .
$$

The velocity of the quasiparticles is given by $\partial \epsilon / \partial p$ which leads to the definition of the effective mass

$$
\frac{1}{m}=\left.\frac{\frac{1}{m_{0}}+\frac{1}{p} \partial_{p} \sigma}{1-\partial_{\omega} \sigma}\right|_{\omega=\epsilon_{f}, p=p_{f}}
$$

where the momenta and energy are set to the Fermi momenta and energy after derivatives. Consequently the quasiparticle energy can be approximated by $\epsilon \approx \frac{p^{2}}{2 m}+\Delta$ with the effective mass (17) and the energy shift $\Delta=$ $\sigma\left(p_{f}, \epsilon_{f}\right)$. This approximation has to be replaced by thermal averaging when finite temperature systems are considered. A useful method would also be to read off the quasiparticle parameterization from current parameterizations of the momentum distribution 43.44 .

Using the definition of the compressibility one obtains directly from (15)

$$
\begin{aligned}
\mathcal{K}=\frac{1}{n^{2}} \frac{\partial n}{\partial \mu}= & -\frac{\beta}{n^{2}} \int \frac{d p}{(2 \pi)^{3}} F(p)(1-F(p)) \\
& \times\left(\left[\Delta^{\prime}(n)-\frac{p^{2}}{2 m}(\ln m)^{\prime}\right] \frac{\partial n}{\partial \mu}-1\right) \\
= & \frac{\mathcal{K}_{0}}{1+n^{2} \frac{\delta}{\delta n} \Delta \mathcal{K}_{0}-\frac{3}{2} \frac{\partial \ln m}{\partial \ln n}}
\end{aligned}
$$

where the free compressibility is $\mathcal{K}_{0}=\frac{\beta}{n^{2}} \int \frac{d p}{(2 \pi)^{3}} F(p)(1-$ $F(p)$ ). Alternatively, the energy shift $\Delta$ and the effective mass can be expressed by Landau parameter.

In the next chapter we will present a consistent dynamical response function for the quasiparticle picture such that the correlations are parameterized by quasiparticles with an effective mass, an energy shift and a relaxation time. From this we will obtain the correct compressibility (18) from the dynamical response via the static limit obeying the frequency sum rules. Actually, a static local field can be constructed provided we choose the effective mass appropriately. This will lead to a recipe how the effective mass can be determined from the structure factor which is well known from Monte-Carlo simulations or experiments.

The conventional compressibility sum rule 45 reads with $\lim _{q \rightarrow 0} \Pi=-n^{2} \mathcal{K}_{0}$ and (6) 


$$
\begin{aligned}
-\lim _{q \rightarrow 0} \frac{2}{\pi V_{q}} \int_{0}^{\infty} \frac{d \omega^{\prime}}{\omega^{\prime}} \operatorname{Im} \epsilon\left(\omega^{\prime}\right) & =\lim _{q \rightarrow 0} \operatorname{Re} \frac{\Pi(0, q)}{1-f_{q}(0) \Pi(0, q)} \\
& =-\lim _{q \rightarrow 0} \frac{n^{2} \mathcal{K}_{0}}{1+f_{q}(0) n^{2} \mathcal{K}_{0}} \\
& \equiv-n^{2} \mathcal{K}
\end{aligned}
$$

such that we can expect from the correct result (18) that the static local field has the form

$$
\lim _{q \rightarrow 0} f_{q}(0)=\frac{\partial \Delta}{\partial n}-\frac{3}{2 n^{2} \mathcal{K}_{0}} \frac{\partial \ln m}{\partial \ln n} .
$$

We will present a dynamical local field which leads in the static limit exactly to this desired result (20).

\section{B. Frequency sum rules}

The sum rules can be easily read off from the fact that the response function is an analytical function in the upper half plane and falls off with large frequencies faster than $1 / \omega^{2}$ such that the compact Kramers Kronig relation reads

$$
\int d \omega^{\prime} \frac{\chi\left(\omega^{\prime}\right)}{\omega^{\prime}-\omega+i 0}=0
$$

closing the contour of integration in the upper half plane. From this one has

$$
\begin{aligned}
\operatorname{Re} \chi(\omega) & =\int \frac{d \omega^{\prime}}{\pi} \frac{\operatorname{Im} \chi\left(\omega^{\prime}\right)}{\omega^{\prime}-\omega} \\
& =\frac{<\omega>}{\omega^{2}}+\frac{<\omega^{3}>}{\omega^{4}}+\ldots
\end{aligned}
$$

with the moments

$$
<\omega^{2 k+1}>=\int \frac{d \omega}{\pi} \omega^{2 k+1} \operatorname{Im} \chi(\omega) .
$$

The first two moments are known exactly to be (appendix (C)

$$
<\omega>=\int \frac{d \omega}{\pi} \omega \operatorname{Im} \chi(\omega)=\frac{n q^{2}}{m}
$$

with the density $n$ and the mass $m$ of the particles and 1 团 8

$$
\begin{aligned}
<\omega^{3}>= & \int \frac{d \omega}{\pi} \omega^{3} \operatorname{Im} \chi(\omega)=2 E_{\text {int }} \frac{q^{4}}{m^{2}}+\frac{n q^{6}}{4 m^{3}} \\
& -\frac{n^{2} q^{4}}{m^{2}} V_{q} \tilde{I}(q) .
\end{aligned}
$$

Here $E_{\text {int }}$ is the kinetic energy of the interacting system and

$$
\begin{aligned}
\tilde{I}(q) & =-\frac{1}{n} \int \frac{d k}{(2 \pi)^{3}}\left(S_{k-q}-S_{k}+n \delta_{k, q}-n \delta_{k, 0}\right) \frac{(k \cdot q)^{2}}{q^{4}} \frac{V_{k}}{V_{q}} \\
& =I(q)-1
\end{aligned}
$$

where $I(q)$ is usually presented in literature明

$$
I(q)=-\frac{1}{n} \int \frac{d k}{(2 \pi)^{3}}\left(S_{k-q}-S_{k}\right) \frac{(k \cdot q)^{2}}{q^{4}} \frac{V_{k}}{V_{q}} .
$$

and $S_{k}$ is the structure factor (10).

In order to understand the different contributions, the short distance limit (C26) from (26) is performed

$$
\begin{aligned}
& \lim _{q \rightarrow \infty} \tilde{I}(q)=-\frac{1}{n} \int \frac{d k}{(2 \pi)^{3}}\left(S_{k}-1+n \delta_{k, 0}\right) \\
& \times {\left[\frac{((k+q) \cdot q)^{2}}{q^{4}} \frac{V_{k+q}}{V_{q}}-\frac{(k \cdot q)^{2}}{q^{4}} \frac{V_{k}}{V_{q}}\right] } \\
&=\left(1-g_{0}\right)+\lim _{q \rightarrow \infty} \frac{1}{n} \int \frac{d k}{(2 \pi)^{3}}\left(S_{k}-1\right) \frac{(k \cdot q)^{2}}{q^{4}} \frac{V_{k}}{V_{q}}-1
\end{aligned}
$$

where the last term comes from the $\delta_{k, 0}$ term The first term alone is sometimes called exact result t. 10 , which holds only for static local fields. The second term describes the motion of particles inside the correlation hole and takes for foulomb just $-\frac{1}{3}\left(1-g_{0}\right)$ which has been pointed out ing. Together one obtains the small distance result

$$
\lim _{q \rightarrow \infty} \tilde{I}(q)=\frac{2}{3}\left(1-g_{0}\right)-1
$$

in agreement with the direct expansion (C26).

Now we proceed and derive the boundaries for the local field $f_{q}(\omega)$ from (雨) by the above sum rules. Therefore we look at the large $\omega$ expansion of (4) from which we can check with the help of (22) the desired sum rules (24) and (25). The simple RPA leads to [see also (47)-(50)]

$$
\Pi_{0}(\omega)=\frac{n q^{2}}{m \omega^{2}}+\left(2 E \frac{q^{4}}{m^{2}}+\frac{n q^{6}}{4 m^{3}}\right) \frac{1}{\omega^{4}}+o\left(1 / \omega^{5}\right),
$$

from which one gets with (4)

$$
\begin{aligned}
& \chi(\omega)=\frac{n q^{2}}{m \omega^{2}}+\left(2 E \frac{q^{4}}{m^{2}}+\frac{n q^{6}}{4 m^{3}}+\frac{n^{2} q^{4}}{m^{2}}\left(V_{q}+f_{q}(\infty)\right)\right) \frac{1}{\omega^{4}} \\
& +o\left(1 / \omega^{5}\right) .
\end{aligned}
$$

The first order energy weighted sum rule (24) is fulfilled trivially provided $\Pi$ fulfills it. The third order sum rule (25) can be fulfilled if we construct the local field according tol

$$
\begin{aligned}
f_{q}(\infty) & =-V_{q}(1+\tilde{I}(q))-\frac{2}{n^{2}}\left(E-E_{\text {int }}\right) \\
& =-V_{q} I(q)-\frac{2}{n^{2}}\left(E-E_{\text {int }}\right)
\end{aligned}
$$

where $E$ is the kinetic energy of the noninteracting system. The last term describes the fact that the third order frequency sum rule of the polarization function yields the noninteracting kinetic energy. This form neglecting the last term has been discussed int. In the later derivation 
of the polarization function we cannot consider the kinetic energy anymore as interaction free, since the relaxation time appears as well as the effective mass. Therefore within the quasiparticle picture used here the difference $E-E_{\text {int }}$ vanishes or positively stated, is accounted for by the effective mass. To facilitate the comparison with the literature we have kept this difference formal as $\Delta E=-\frac{2}{n^{2}}\left(E-E_{\text {int }}\right)$.

With $(\sqrt{32})$ we have given the constraint on the dynamical local field from the third order frequency sum rule. In the following we will present a dynamical local field which fulfills both requirements, the compressibility (20) and the frequency sum rule (32).

\section{DYNAMICAL RESPONSE FUNCTION}

In 42 was given the polarization function for an interacting quantum system imposing conservation laws on the relaxation time approximation. These polarization functions we have denoted by $\Pi^{\mathrm{n}}$ for density conservation imposed, $\Pi^{\mathrm{n}, \mathrm{j}}$ for density and current conservation and $\Pi^{\mathrm{n}, \mathrm{j}, \mathrm{E}}$ for density, current and energy conservation. In the former paper we could give only formal matrix expressions for the response function. In appendix A we repeat shortly the way of derivation from the quantum kinetic theory and give now the explicit form of the response function. We obtain with (A15) and A16

$$
\chi(\omega)=\frac{\Pi^{\mathrm{n}, \mathrm{j}, \mathrm{E}}(\omega)}{1-V_{0} \Pi^{\mathrm{n}, \mathrm{j}, \mathrm{E}}(\omega)-2 m V_{4} \Pi_{13}(\omega)}
$$

where

$$
\begin{aligned}
\Pi_{13}(\omega) & =\frac{\Pi^{\mathrm{n}, \mathrm{j}, \mathrm{E}}}{2 m} \frac{\Pi_{h} \Pi_{2}(0)-i \tau \omega \Pi_{2}\left(\omega+\frac{i}{\tau}\right)}{\Pi_{h} \Pi_{0}(0)-i \tau \omega \Pi_{0}\left(\omega+\frac{i}{\tau}\right)} \\
\Pi_{h} & =\frac{\Pi_{2}^{2}\left(\omega+\frac{i}{\tau}\right)-\Pi_{0}\left(\omega+\frac{i}{\tau}\right) \Pi_{4}\left(\omega+\frac{i}{\tau}\right)}{\Pi_{2}^{2}(0)-\Pi_{0}(0) \Pi_{4}(0)}
\end{aligned}
$$

and

$$
\begin{aligned}
& V_{0}=\frac{\delta}{\delta n} \Delta-V_{4} \frac{q^{2}}{4}+V_{q} \\
& V_{4}=\frac{\delta}{\delta n} \frac{1}{2 m}=-\frac{1}{2 n m} \frac{\partial \ln m}{\partial \ln n} .
\end{aligned}
$$

The explicit expressions of the moments of the polarization function are summarized in appendix B for computation. From the response function (33) we will read off the main result of this paper: the dynamical local field for quasiparticles with the effective mass and energy. Now we are going to work out explicitly the form of local field to show that the third order sum rule can be fulfilled and the correct compressibility is obtained.

\section{A. Dynamical local field}

Comparing (33) with (4) an intermediate dynamical local field can be read off as $\left[V_{0}=\frac{\delta}{\delta n} \Delta-V_{4} q^{2} / 4+V_{q}\right]$

$$
\begin{aligned}
\tilde{f}_{q}(\omega) & =\frac{\delta}{\delta n} \Delta+V_{4}\left(\frac{\Pi_{h} \Pi_{2}(0)-i \tau \omega \Pi_{2}\left(\omega+\frac{i}{\tau}\right)}{\Pi_{h} \Pi_{0}(0)-i \tau \omega \Pi_{0}\left(\omega+\frac{i}{\tau}\right)}-\frac{q^{2}}{4}\right) \\
& =\frac{\delta}{\delta n} \Delta+V_{4}\left(\frac{\Pi_{2}(0)}{\Pi_{0}(0)}-\frac{q^{2}}{4}\right)+o\left(\frac{1}{\omega}\right) \\
& =\frac{\delta}{\delta n} \Delta-\frac{\partial \ln m}{\partial \ln n}\left\{\begin{array}{l}
\frac{3}{2 n^{2} K_{0}}+o\left(q^{2}\right) \\
\frac{1}{3} \frac{E}{n^{2}}+o\left(1 / q^{2}\right)
\end{array}+o\left(\frac{1}{\omega}\right)\right.
\end{aligned}
$$

where we used the expansion of appendix B, explicitly (B16), in the first line and (B14) and (B15) in the last line. Please remark that $\Pi^{\mathrm{n}, \mathrm{j}}, \mathrm{E}$ itself contains correlations beyond the polarization in RPA, $\Pi_{0}$. This we will present in a moment.

First, we see the astonishing result that obviously

$$
\lim _{\omega \rightarrow 0} \tilde{f}_{q}(\omega)=\lim _{\omega \rightarrow \infty} \tilde{f}_{q}(\omega)
$$

the static local field required for the compressibility agrees with the infinite frequency limit required for the third order sum rule (31). F This shows that the answer to the sumrule puzzle is not due to the explicit dynamical character of the local field as often claimed in the literature. Instead we will see in paragraph IIID that it is due to the underlying selfenergy correction which has to obey certain relations.

At this point it is important to avoid a misunderstanding. The intermediate dynamical local field, $\tilde{f}_{q}$, is not the total one describing correlations beyond RPA polarization function, $\Pi_{0}$, which would be the case only in the infinite frequency limit. Instead, part of the correlations are already captured in $\Pi^{\mathrm{n}, \mathrm{j}, \mathrm{E}}$. To make this explicit we write 33 as

$$
\begin{aligned}
\frac{1}{\chi(\omega)} & =\frac{1}{\Pi^{\mathrm{n}, \mathrm{j}, \mathrm{E}}(\omega)}-V_{q}-\tilde{f}_{q}(\omega) \\
& =\frac{1}{\Pi_{0}(\omega)}-V_{q}-\tilde{f}_{q}(\omega)+\tilde{f}_{q}^{*}(\omega)
\end{aligned}
$$

where the difference between $\Pi^{\mathrm{n}, \mathrm{j}, \mathrm{E}}$ and $\Pi_{0}$ has been recasted into a local field contribution 16 derived from (A12)- A14)

\footnotetext{
*One should not be mislead to the conclusion that this violates the Kramers Kronig relation for $\tilde{f}_{q}(\omega)$. A toy example of $\operatorname{Im} \tilde{f}=\sin (a \omega) /\left(\omega^{2}-4 \pi^{2} / a^{2}\right)$ and the corresponding real part obtained from the Kramers Kronig relation (22) shows that indeed (37) can hold simultaneously with the Kramers Kronig relation.
} 


$$
\begin{aligned}
\tilde{f}_{q}^{*}(\omega) & =\frac{1}{\Pi^{\mathrm{n}, \mathrm{j}, \mathrm{E}}(\omega)}-\frac{1}{\Pi_{0}(\omega)} \\
& =-\frac{1}{1-i \omega \tau}\left(\frac{1}{\partial_{\mu} n}-\frac{2 E}{n^{2}}\right)+o\left(q^{2}\right) \\
& =\frac{1}{1-i \omega \tau} \frac{8 \epsilon_{f}}{15 n}+o\left(q^{4}\right)
\end{aligned}
$$

with the last line valid for zero temperature. Oppositely in the static limit $\tilde{f}_{q}^{*}(0)=0$. Together with (36) we obtain an effective local field renormalizing the RPA

$$
\begin{aligned}
f_{q}^{\mathrm{eff}}(\omega) & =\tilde{f}_{q}(\omega)-\tilde{f}_{q}^{*}(\omega) \\
& =\frac{\delta}{\delta n} \Delta-\frac{\partial \ln m}{\partial \ln n} \frac{3}{2 n^{2} K_{0}}+\frac{\frac{1}{\partial_{\mu} n}-\frac{2 E}{n^{2}}}{1-i \omega \tau}+o\left(q^{2}\right) \\
& =\frac{\delta}{\delta n} \Delta-\frac{\epsilon_{f}}{n}\left(\frac{\partial \ln m}{\partial \ln n}+\frac{1}{1-i \omega \tau} \frac{8}{15}\right)
\end{aligned}
$$

where the last line is again the zero temperature limit. The high frequency limit required for the third order sum rule all agree $f_{q}^{\text {eff }}(\infty)=\tilde{f}_{q}(\infty)=f_{q}(\infty)$.

\section{B. Connection to density functionals}

In order to establish the connection to the ground state exact relations 47 of exchange correlation energy, $\varepsilon_{\mathrm{xc}}$, we see from (40)

$\lim _{\omega \rightarrow 0} \lim _{q \rightarrow 0} f_{q}^{\mathrm{eff}}(\omega) \equiv \frac{d^{2}}{d n^{2}}\left[n \varepsilon_{\mathrm{xc}}(n)\right]=\frac{\delta}{\delta n} \Delta-\frac{\epsilon_{f}}{n}\left(\frac{\partial \ln m}{\partial \ln n}+\frac{8}{15}\right)$

and

$$
\lim _{q \rightarrow 0} f_{q}^{\mathrm{eff}}(\infty) \equiv-\frac{4}{5} n^{2 / 3} \frac{d}{d n}\left[\frac{\varepsilon_{\mathrm{xc}}(n)}{n^{2 / 3}}\right]=\frac{\delta}{\delta n} \Delta-\frac{\epsilon_{f}}{n} \frac{\partial \ln m}{\partial \ln n}
$$

with $f_{0}^{\text {eff }}(0)<f_{0}^{\text {eff }}(\infty)<0$. This establishes the link to time dependent density functional theories 4850 . A overview about different approximation schemes are given in $\$$. We will see in paragraph IIID that in order to fulfill the third order frequency sum rule we have to have an additional effective mass beyond $\varepsilon_{\mathrm{xc}}$.

\section{Compressibility sum rule}

From (36) one sees immediately that the required form for the compressibility sum rule (20) appears, since $\Pi^{\mathrm{n}, \mathrm{j}, \mathrm{E}}(0)=\Pi_{0}(0)$. Therefore we have derived a dynamical response function and a local field which shows in the static limit the correct compressibility (18). This compressibility formulae (18) can be checked alternatively by calculating explicitly the frequency integral in (19). The required small wavevector limit of the so called screened structure function (19) takes the form $\left[\Pi=\Pi^{\mathrm{n}, \mathrm{j}, \mathrm{E}}\right]$

$$
\begin{aligned}
& \frac{n}{\beta} K=\lim _{q \rightarrow 0} \frac{1}{n \pi} \int \frac{d \omega}{1-\mathrm{e}^{-\beta \omega}} \\
& \times \frac{\operatorname{Im} \Pi(\omega)\left(1-\bar{f}_{q}(\omega) \operatorname{Re} \Pi(\omega)\right)+\operatorname{Im} \Pi(\omega) \bar{f}_{q}(\omega) \operatorname{Re} \Pi(\omega)}{\left(1-\bar{f}_{q}(\omega) \operatorname{Re} \Pi(\omega)\right)^{2}+\left(\bar{f}_{q}(\omega) \operatorname{Im} \Pi(\omega)\right)^{2}}
\end{aligned}
$$

where $\tilde{f}_{q}(\omega)=\bar{f}_{q}(\omega)-V_{q}$. Now we observe that

$$
\begin{aligned}
& \lim _{q \rightarrow 0} \operatorname{Im} \Pi(\omega)=\lim _{q \rightarrow 0} \operatorname{Im}_{0}(\omega) \\
& =-\pi \int \frac{d p}{(2 \pi)^{3}}\left(\mathrm{e}^{-\beta \omega}-1\right) \delta\left(\omega-\frac{p \cdot q}{m}\right) F(p)(1-F(p))+o\left(q^{2}\right) \\
& =0+o\left(q^{2}\right)
\end{aligned}
$$

vanishes for small $q$. Therefore we have to perform the limit in (43) in the distribution sense to obtain

$$
\begin{aligned}
\frac{n}{\beta} K & =\lim _{q \rightarrow 0} \frac{1}{n \pi} \int \frac{d \omega}{1-\mathrm{e}^{-\beta \omega}} \\
& \times\left(\frac{\operatorname{Im} \Pi_{0}(\omega)}{1-\bar{f}_{q} \operatorname{Re} \Pi(\omega)}+\pi \operatorname{Re} \Pi(\omega) \delta\left(1-\bar{f}_{q} \operatorname{Re} \Pi(\omega)\right)\right) .
\end{aligned}
$$

It is not difficult to see that the second part vanishes and we obtain

$$
\begin{aligned}
\frac{n}{\beta} K & =\frac{1}{n} \frac{\int \frac{d p}{(2 \pi)^{3}} F(p)(1-F(p))}{1-\bar{f}_{q} \operatorname{Re}(0)} \\
& =\frac{1}{n \beta} \frac{n^{2} K_{0}}{1+n^{2} K_{0} \frac{\partial \Delta}{\partial n}-\frac{3}{2} \frac{\partial \ln m}{\partial \ln n}}
\end{aligned}
$$

which agrees with (18).

Therefore, we have shown that the dynamical local field (36) from the response function (33) leads to the same compressibility (19). This gives besides the compressibility sum rule already checked a second proof that we have derived a dynamical local field which leads to the correct compressibility. The static limit will allow now to complete compressibility and third order frequency sum rule simultaneously.

\section{Frequency sum rules}

Now that we have the response function (33) at hand we can proceed and proof the frequency sum rules (24) and (25) explicitly. First we expand the polarization functions for large frequencies. The superscript denotes which conservation laws are obeyed, density $(n)$, energy $(E)$ and current $(j)$ respectively. We obtain

$$
\Pi^{\mathrm{n}}(\omega)=\frac{n q^{2}}{m \omega^{2}}-i \frac{n q^{2}}{m \omega^{3} \tau}+\left(2 E \frac{q^{4}}{m^{2}}+\frac{n q^{6}}{4 m^{3}}-\frac{n q^{2}}{m \tau^{2}}\right) \frac{1}{\omega^{4}}
$$




$$
\begin{aligned}
& -i \frac{q^{4}}{m^{2} \omega^{5} \tau}\left(\frac{n^{2}}{\Pi_{0}(0)}+6 E-\frac{n m}{q^{2} \tau^{2}}+\frac{3 n q^{2}}{4 m}\right)+o\left(\frac{1}{\omega^{6}}\right) \\
& \Pi^{\mathrm{n}, \mathrm{E}}(\omega)=\Pi^{\mathrm{n}}(\omega)+o\left(\frac{1}{\omega^{5}}\right) \\
& \Pi^{\mathrm{n}, \mathrm{j}}(\omega)=\frac{n q^{2}}{m \omega^{2}}+\left(2 E \frac{q^{4}}{m^{2}}+\frac{n q^{6}}{4 m^{3}}\right) \frac{1}{\omega^{4}} \\
& -i \frac{q^{4}}{m^{2} \omega^{5} \tau}\left(\frac{n^{2}}{\Pi_{0}(0)}+2 E+\frac{n q^{2}}{4 m}\right)+o\left(\frac{1}{\omega^{6}}\right) \\
& \Pi^{\mathrm{n}, \mathrm{j}, \mathrm{E}}(\omega)=\Pi^{\mathrm{n}, \mathrm{j}}(\omega)+o\left(\frac{1}{\omega^{5}}\right) .
\end{aligned}
$$

We see that the current conservation repairs some defiances of the Mermin-Das polarization function, $\Pi^{\mathrm{n}}$ which obeys only density conservation, in that the imaginary part shows a different frequency behavior

$$
\begin{aligned}
\lim _{\omega \rightarrow \infty} \operatorname{Im} \Pi^{\mathrm{n}, \mathrm{j}, \mathrm{E}}(q, \omega) & =\frac{n^{2} q^{4}}{\omega^{5} \tau m^{2}}\left(\frac{1}{\partial_{\mu} n}-\frac{2 E}{n^{2}}\right) \\
\lim _{\omega \rightarrow \infty} \operatorname{Im}^{\mathrm{n}}(q, \omega) & =-\frac{n q^{2}}{\omega^{3} \tau m} .
\end{aligned}
$$

The last formulae corrects a misprint in formula (23) oft6. This different behavior of the imaginary part is also reflected in different expressions for the third order moment $\left(o\left(1 / \omega^{4}\right)\right)$ or third order sum rule.

From (22) and (47)-(50) we read off the sum rules

$$
<\omega>=\int \frac{d \omega}{\pi} \omega \operatorname{Im} \Pi(\omega)=\frac{n q^{2}}{m}
$$

which holds for each $\Pi^{\mathrm{n}}, \Pi^{\mathrm{n}, \mathrm{j}}, \Pi^{\mathrm{n}, \mathrm{j}, \mathrm{E}}$ and $\chi$. In contrast to that we will see now that the third order sum rule gives different results for the inclusion of different conservations laws. Using the polarization function including density, energy and momentum conservation we obtain from 49.

$$
<\omega^{3}>=\int \frac{d \omega}{\pi} \omega^{3} \operatorname{Im} \Pi^{\mathrm{n}, \mathrm{j}, \mathrm{E}}(\omega)=2 E \frac{q^{4}}{m^{2}}+\frac{n q^{6}}{4 m^{3}} .
$$

We remark that according to (47)-(50) the Mermin-Das polarization (A14) including only density conservation or even including additionally energy conservation (A13) would yield an additional $-n q^{2} / m \tau^{2}$ term which is an artefact. This is repaired by additionally taking into account momentum conservation.

Comparing (25) with (54) we see that just the last terms are missing. In order to obtain this sum rule we have to use the response function (33) and not the polarization function for which this sum rule is actually designed. With (36) and (31) one gets

$$
\chi(\omega)=\Pi^{\mathrm{n}, \mathrm{j}, \mathrm{E}}(\omega)+\frac{n^{2} q^{4}}{m^{2}}\left(V_{0}+\frac{\Pi_{2}(0)}{\Pi_{0}(0)} V_{4}\right) \frac{1}{\omega^{4}}+o\left(\frac{1}{\omega^{5}}\right) .
$$

Consequently, the third order sum rule 32 is rendered correctly if one sets

$$
\begin{aligned}
f_{q}(\infty) & =V_{0}-V_{q}+V_{4} \frac{\Pi_{2}(0)}{\Pi_{0}(0)}=\frac{\delta \Delta}{\delta n}+V_{4}\left(\frac{\Pi_{2}(0)}{\Pi_{0}(0)}-\frac{q^{2}}{4}\right) \\
& \equiv-V_{q}(1+\tilde{I}(q))+\Delta E .
\end{aligned}
$$

By the requirement (56) we have a possibility to fulfill the third order sumrule exactly from the dynamical response as well as static local field model.

\section{E. Consequences on selfenergies}

Let us now work out what that means for our selfenergy parameterization $\Delta$ and $m$. From (56) we obtain a determining condition for the effective mass and energy shift $[I(q)=1+\tilde{I}(q)]$

$$
V_{q} I(q)=\frac{1}{2 m n}\left(\frac{\Pi_{2}(0)}{\Pi_{0}(0)}-\frac{q^{2}}{4}\right) \frac{\partial \ln m}{\partial \ln n}-\frac{\delta \Delta}{\delta n}+\Delta E .
$$

Since we work with the effective mass and shift parameterization of the quasiparticle energy the difference between $E$ and $E_{\text {int }}, \Delta E$, vanishes but we keep it for completeness further on.

Applying the small wavevector limit $(\mathrm{C} 25)$ and $(36)$ we see now from (57) for Coulomb systems

$$
\lim _{q \rightarrow 0}\left(\frac{\delta \Delta}{\delta n}-\frac{3}{2} \frac{1}{n^{2} K_{0}} \frac{\partial \ln m}{\partial \ln n}\right)=\Delta E-\frac{2}{5} \frac{q^{2} V_{q}}{k_{f}^{2}} \gamma+o\left(q^{2}\right)
$$

Oppositely from (57), the large wavevector or small distance limit (C26) and (36)reads

$$
\lim _{q \rightarrow \infty}\left(\frac{\delta \Delta}{\delta n}-\frac{1}{3} \frac{E}{n^{2}} \frac{\partial \ln m}{\partial \ln n}\right)=\Delta E-\frac{2}{3}\left(1-g_{0}\right) V_{q}+o\left(1 / q^{2}\right)
$$

where the last term on the right side vanishes for Coulomb potentials and persists only for potentials which range falls faster than Coulomb.

If we assume homogeneous systems, where $\Delta$ and $m$ becomes independent of the wavevector, the equations (58) and (59) determines the quasiparticle shift as well as the effective mass via

$$
\begin{aligned}
\frac{\partial \ln m}{\partial \ln n} & =\frac{\frac{2 q^{2} V_{q}}{5 k_{f}^{2}} \gamma}{\frac{3}{2} \frac{1}{n^{2} K_{0}}-\frac{1}{3} \frac{E}{n^{2}}} \\
\frac{\delta \Delta}{\delta n} & =\frac{\frac{2 q^{2} V_{q}}{5 k_{f}^{2}} \gamma}{\frac{9}{2} \frac{1}{E K_{0}}-1}+\Delta E-\frac{2}{3}\left(1-g_{0}\right) V_{q}+o\left(1 / q^{2}\right)
\end{aligned}
$$

where the last term vanishes for Coulomb potentials. If we remember the relation between the selfenergy $\sigma$ and 
the effective mass (17) we can determine from (60) the thermal averaged selfenergy and wave function renormalization $\mathcal{Z}=\left(1-\partial_{\omega} \sigma\right)^{-1}$. For the special case of zero temperature and neglecting the usually small $\partial_{p} \sigma$ part, it reads

$$
\begin{gathered}
\Delta=\left.\sigma\right|_{\epsilon=\epsilon_{f}}=\frac{1}{10} q^{2} V_{q} \int_{0}^{n} d n^{\prime} \frac{\gamma\left(n^{\prime}\right)}{p_{f}^{2}\left(n^{\prime}\right)}+\int_{0}^{n} d n^{\prime} \Delta E \\
\left.\ln \mathcal{Z}\right|_{\epsilon=\epsilon_{f}}=-m q^{2} V_{q} \int_{0}^{n} d n^{\prime} \frac{\gamma\left(n^{\prime}\right)}{p_{f}^{4}\left(n^{\prime}\right)}
\end{gathered}
$$

with $\gamma$ from (9) such that the effective mass takes the form

$$
m=m_{0} \exp \left\{m q^{2} V_{q} \int_{0}^{n} d n^{\prime} \frac{\gamma\left(n^{\prime}\right)}{p_{f}^{4}\left(n^{\prime}\right)}\right\} .
$$

The general expression for finite temperatures is given by (60).

Since the needed expressions $\tilde{I}$ in (26) or $\gamma$ in (9) are functions of the structure function which is given itself again by the response function $(10)$ we have the usual self-consistent procedure analogous to (\$) first introduced by Singwi Sjölander 4 but with another $G(q)$. Here we suggest to obtain the effective mass $m$ and energy shift by (57) using $I(q)$.

Alternatively one might use the well known experimental values of the static structure factor $S_{q}$ and determine by this way the effective mass and energy shift. This definition of effective mass has the advantage that the third order and compressibility sum rule of the response function will be rendered exactly. Therefore equation (60) is the second main result of this paper.

Let us remark that if we would have no effective mass but a mere density dependent selfenergy $\sigma$ like in density functional theories, the requirement of (58) and (59) corresponding to the compressibility and third order frequency sum rule cannot be fulfilled simutaneously. This was remarked in detail in literature 11 15. By including the effective mass we can resolve this puzzle here.

\section{NUMERICAL RESULTS AND DISCUSSION}

As a test example we will now consider the unpolarized electron gas in three dimensions (3D) at zero temperature. The density parameter is the usual Bruckner parameter, defined as the ratio of interparticle distance to the Bohr radius, $r_{s}=(3 / 4 \pi n)^{1 / 3} / a_{0}$. First we will give a simple quasiparticle picture (QP), where the $\Delta$ and the effective mass are determined as density-dependent constants from MC data. In the second step, we will allow that the $\Delta$ and the effective mass depend on the wavevector. This will lead to the self-consistent quasiparticle picture (SQP).
In order to calculate the quasiparticle parameter $\Delta$ and the effective mass, of mploy the results of Monte Carlo (MC) simulations23.24. For a parameterization of the $\mathrm{MC}$ data see 2 2. In 1 was discussed the difference of interacting and free kinetic energies, $\Delta E=\frac{2}{n^{2}}\left(E_{\text {int }}-E\right)$. This difference is given as $\delta=E_{\text {int }} / E-1$ by the MC data of Ceperley and Alder. In our quasiparticle picture one has $E_{\text {int }} / E=m_{0} / m$, and the effective mass is given by $m=m_{0} /(1+\delta)$. This allows us to determine the needed derivative as

$$
\frac{\partial \ln m}{\partial \ln n}=\frac{r_{s}}{3(1+\delta)} \frac{\partial \delta}{\partial r_{s}} .
$$

\begin{tabular}{|l||c|c|c||c|c|c|}
\hline \hline \multicolumn{1}{c||}{$r_{s}$} & $\gamma_{0}$ & $\delta$ & \multicolumn{4}{c|}{$1-g(0)$} \\
& $\mathrm{MC}$ & $\mathrm{MC}$ & $\mathrm{MC}$ & $\mathrm{SQP}$ & $\mathrm{QP}$ & $\mathrm{PV}+\Delta \mathrm{E}$ \\
\hline 1 & 0.2567 & 0.036 & 0.7276 & 0.7240 & 0.6626 & 0.6971 \\
2 & & 0.091 & $(0.8627)$ & 0.8627 & 0.7666 & 0.8346 \\
3 & 0.2722 & & 0.9078 & 0.9201 & 0.8456 & \\
5 & 0.2850 & 0.292 & 0.9768 & 0.9627 & 0.9351 & 1.0558 \\
10 & 0.3079 & 0.619 & 0.9976 & 0.9733 & 0.9613 & 1.1231 \\
\hline \hline
\end{tabular}

TABLE I. The long wavelength limit of the local field factor, $\gamma_{0}$, and the small range value of the pair correlation according to the MC data o 23 . The value in brackets is an interpolation. The quasiparticle (QP), self-consistent quasiparticle (SQP) as well as Pathak-Vashishta value together with $\Delta E$ $(\mathrm{PV}+\Delta E)$ is given for comparison.

The difference $\Delta E$ is given by the large-wavelength limit of the local field

$$
\begin{aligned}
\lim _{q \rightarrow 0} G(q) & =\gamma_{0}\left(\frac{q}{k_{f}}\right)^{2}=\lim _{q \rightarrow 0} \frac{1}{V_{q}}\left(V_{q} I(q)-\Delta E\right) \\
& =\lim _{q \rightarrow 0} \frac{1}{V_{q}}\left(\frac{1}{2 m n} \frac{\partial \ln m}{\partial \ln n}-\frac{\delta \Delta}{\delta n}\right),
\end{aligned}
$$

which is presented by $\gamma_{0} 23$, see table This allows us to determine the quasiparticle energy $\frac{\partial \Delta}{\delta n}$, since the last line of (64) is just (57). The first model can be called improved Pathak/Vashishta scheme $(\mathrm{PV}+\Delta E)$ while the second one together with the effective mass (63) establishes the quasiparticle picture proposed here. We remark that, in order to realize a certain Bruckner parameter $r_{s}$, the quasiparticle picture must be calculated with $r_{s}(1+\delta)$, since all formulae work with effective mass leading to $r_{s} /(1+\delta)$. 


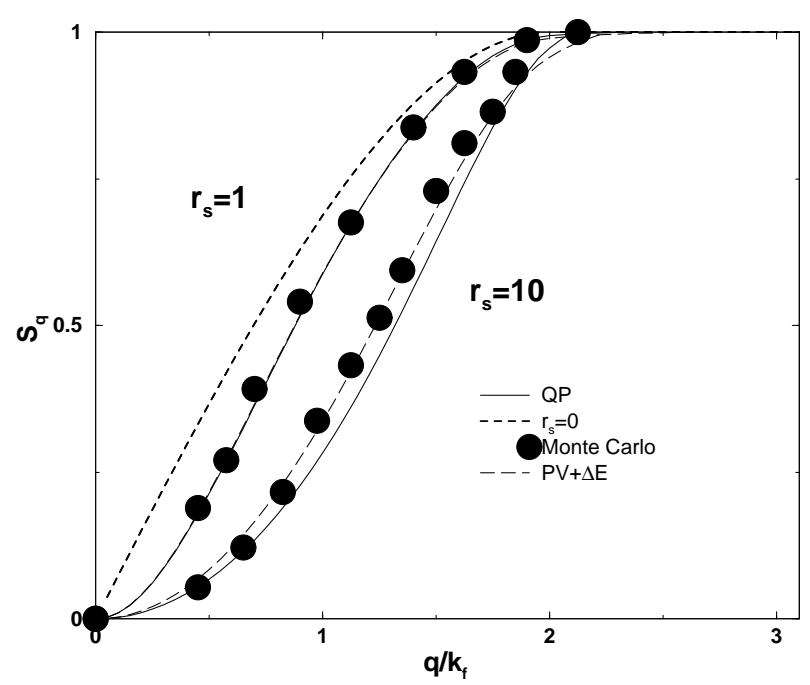

FIG. 1. The static structure factor for $r_{s}=1$ and $r_{s}=10$ from the (PV)- the (PV+ $+E)$ - md (QP)- model compared to the MC data 23 as presented in 25.

With this determination of the QP parameters as well as $(\mathrm{PV}+\Delta E)$ from $\mathrm{MC}$ data we can now compare the results of the iteration scheme $f_{q} \rightarrow S_{q} \rightarrow I(q) \rightarrow f_{q}$. For zero temperature all formulae simplifies and we give them here for convenience in dimensionless momentum $K=q / k_{f}$, energy $\Omega=\omega / \epsilon_{f}$ and distance $R=r k_{f} / \hbar$. Then the dimensionless effective local field is given by

$$
\begin{aligned}
f_{K}= & -\left(\frac{2 s^{4}}{9 \pi^{4}}\right)^{1 / 3} r_{s}\left(\frac{I(K)}{K^{2}}+\gamma_{0}-\frac{2}{5} \gamma\right) \\
& -\frac{r_{s}}{4(1+\delta)} \frac{\partial \delta}{\partial r_{s}}\left(1-\frac{\Pi_{2}(0, K)}{\Pi_{0}(0, K)}+\frac{K^{2}}{4}\right)
\end{aligned}
$$

with $\gamma_{0}$ from (64) and $\gamma=-\frac{1}{s} \int_{0}^{\infty} d K\left(S_{K}-1\right)$ from (9) and the spin degeneracy for electrons $s=2$. The $(\mathrm{PV})$ model would consists only in the term $I(K)$ on the right hand side of (65) and the $(\mathrm{PV}+\Delta E)$ model takes into account the first line of $(65)$. The (QP) picture finally takes all terms into account where we have used (56) or (57) and the requirement (64). The dimensionless function, $\frac{\Pi_{2}(0, K)}{\Pi_{0}(0, K)}=\frac{\Pi_{2}(0)}{k_{f}^{2} \Pi_{0}(0)}$ plotted in figure 5, is given by

$$
\begin{aligned}
& \frac{\Pi_{2}(0, K)}{\Pi_{0}(0, K)}=\frac{1}{2} \frac{3-\frac{K^{2}}{4}-\frac{1}{4 K}\left(1-\frac{K^{2}}{4}\right)^{2} \ln \left|\frac{2-K}{2+K}\right|}{1-\frac{1}{4 K}\left(1-\frac{K^{2}}{4}\right) \ln \left|\frac{2-K}{2+K}\right|} \\
& =\frac{1}{4}\left\{\begin{array}{c}
1-\frac{K^{2}}{3}+o\left(K^{4}\right) \\
\frac{K^{2}}{4}+\frac{1}{5}-\frac{48}{175 K^{2}}+o\left(K^{-4}\right)
\end{array}\right.
\end{aligned}
$$

Provided we know the effective local field, $f_{K}$ in (65), the static structure factor can be obtained from (10) as

$$
S_{K}=\frac{3}{4 \pi} \int_{0}^{\infty} d \Omega \operatorname{Im} \frac{\Pi_{0}(\Omega, K)}{1-\left(f_{K}+\left(\frac{2 s^{4}}{9 \pi^{4}}\right)^{1 / 3} \frac{r_{s}}{K^{2}}\right) \Pi_{0}(\Omega, K)}
$$

where we have used the zero temperature dimensionless quantum polarization from (B1)

$$
\begin{gathered}
\Pi_{0}(\Omega, K)=-1+\frac{4 K^{2}-\left(\Omega-K^{2}\right)^{2}}{8 K^{3}} \ln \frac{2 K+\Omega-K^{2}}{2 K-\Omega+K^{2}} \\
-\frac{4 K^{2}-\left(\Omega+K^{2}\right)^{2}}{8 K^{3}} \ln \frac{2 K+\Omega+K^{2}}{2 K-\Omega-K^{2}} \\
+i \frac{\pi}{2} \begin{cases}0 & \text { for } K<2 \text { and }|\Omega|<\left|K^{2}-2 K\right| \\
\frac{\Omega}{K} & \text { for }\left|K^{2}-2 K\right|<|\Omega|<\left|K^{2}+2 K\right| \\
\frac{4 K^{2}-\left(\Omega-K^{2}\right)^{2}}{4 K^{3}}\end{cases}
\end{gathered}
$$

With the help of the static structure factor we have the pair correlation function (11)

$$
g_{R}=1+\frac{3}{s R} \int_{0}^{\infty} d K K \sin (K R)\left(S_{K}-1\right)
$$

from which the required $I(K)$ function reads according to (C24)

$$
I(K)=-2 \int_{0}^{\infty} \frac{d R}{R}\left(g_{R}-1\right) j_{2}(K R)
$$

with the spherical Bessel function $j_{2}(x)$. This function now enters (65) closing the iteration.

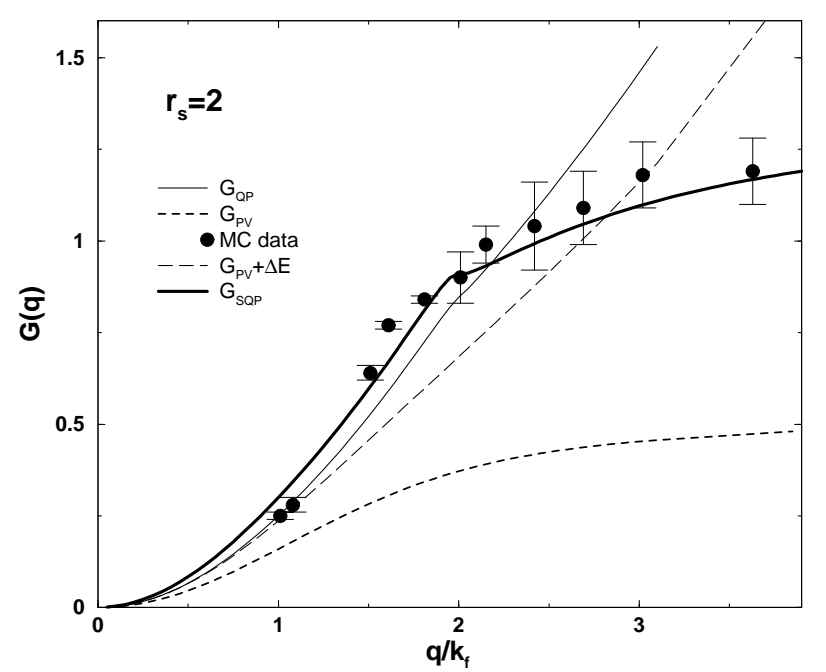

FIG. 2. The static local field for $r_{s}=2$ from the (PV) the $(\mathrm{PV}+\Delta E)$ - and $(\mathrm{QP})-$ model compared to the $\mathrm{MC}$ data24.

In table I we compare the small distance value of the pair correlation (11) of the $(\mathrm{PV}+\Delta E)$ model with the (QP) model. We see that for more dense systems the $(\mathrm{PV}+\Delta E)$ model leads to correlations which are too large while the (QP) model is lower than the $\mathrm{MC}$ values at higher $r_{s}$. In figure 11 we compare the static structure factor of the two models with the MC data. We see that a difference occurs between the $(\mathrm{PV}+\Delta E)$ and $(\mathrm{QP})$ model at higher $r_{s}$. 


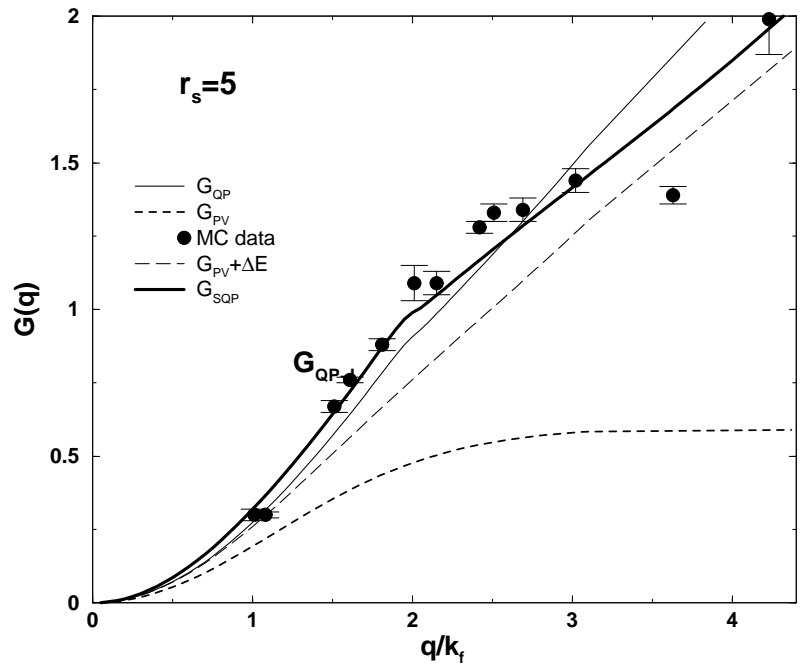

FIG. 3. The static local field for $r_{s}=5$ from the (PV) the $(\mathrm{PV}+\Delta E)$ - and (QP)- model compared to the MC data24.

The difference between the models becomes more apparent if we plot the local field factor as in figures 24.4. We see that the simple (PV) model underestimates the $\mathrm{MC}$ data though it satisfies the third order frequency sum rule. This result is improved by adding the $\Delta E$ read off from the large wavelength limit of MC data. Further improvement is achieved in the $(\mathrm{QP})$ picture. At smaller densities, $r_{s}=2$, the local field is overestimated at higher wavevectors which leads to the deviation seen in table I.

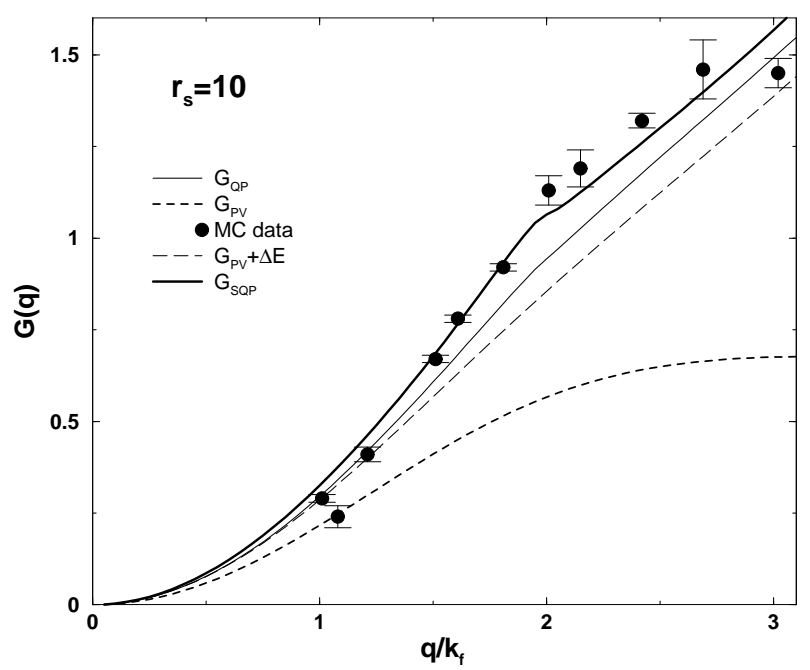

FIG. 4. The static local field for $r_{s}=10$ from the (PV) the $(\mathrm{PV}+\Delta E)$ - and (QP)- model compared to the MC data24.

The (QP) curves show a small hump at $q=2 k_{f}$ in contrast to the $(\mathrm{PV}+\Delta E)$ model. This comes from the function $\frac{\Pi_{2}(0)}{\Pi_{0}(0)}$ which is plotted in figure 5 .

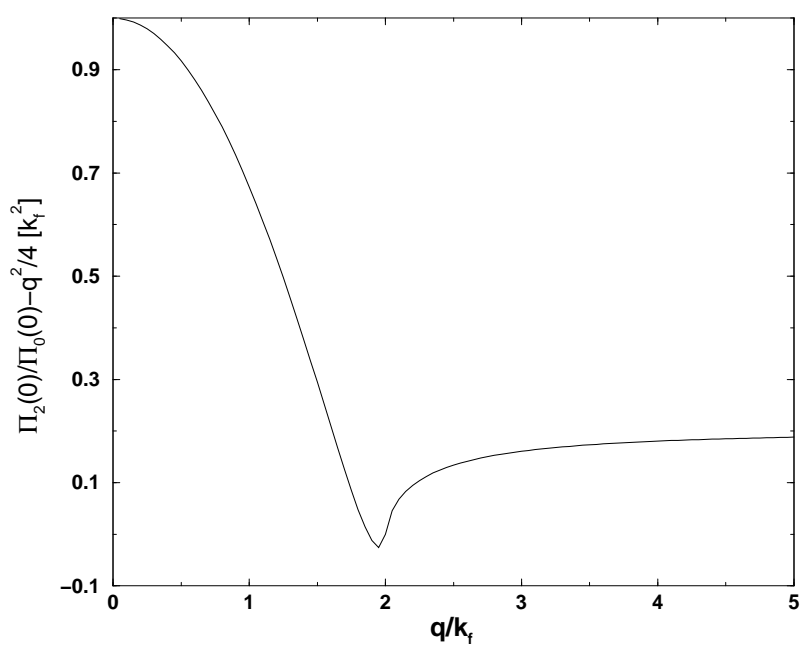

FIG. 5. $\frac{\Pi_{2}(0)}{\Pi_{0}(0)}-\frac{q^{2}}{4}$ of $(57)$ or explicitly (66) for three dimensions at zero temperature.

While in 3D this hump at $q=2 k_{\text {in }}$ is not much pronounced, it shows up in $2 \mathrm{D}$ systems 53 . With the help of the formulae in appendix B one can present also the $2 \mathrm{D}$ results which should be devoted to another paper.

According to (57) the functional form of $\Pi_{2} / \Pi_{0}$ on the right hand side should be equal to $I(k)$ which is a smooth function according to figures 2 4 . Therefore with the simple quasiparticle picture considered so far one cannot satisfy the third order sum rule for all $q$. In order to achieve this, we must allow $\Delta$ as well as the effective mass to carry a $q$-dependence. Of course this leads to a selfconsistent quasiparticle picture, since the energies under integration of the polarization function now change their dispersion. The iteration scheme is therefore enlarged to $f_{q} \rightarrow S_{q} \rightarrow I(q) \rightarrow \Delta(q), m(q) \rightarrow f_{q}$ according to (57). We call this iteration here self-consistent quasiparticle picture (SQP). It satisfies the third order frequency sum rule and the compressibility sum rule simultaneously for all $q$. The results describe the MC data in figures 2 . 4. Also, the small distance value of the pair correlation function is now in better agreement with the MC data as

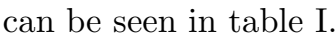

\section{SUMMARY}

We have derived a response function in the quasiparticle picture where the correlations are parameterized by a density-dependent effective mass, energy shift and a relaxation time respecting density, energy and momentum conservation. The dynamical response function can be given in the form of a modified RPA including a dynamical local field. This local field leads in the static limit to the correct compressibility. The effective mass and quasiparticle energy shift are proposed to be determined by the requirement of the third order sum rule. This allows in turn to satisfy the compressibility sum rule simultaneously. Since the effective mass is now a function of 
the structure factor, one might use experimentally known values or simulation results to construct a more realistic quasiparticle picture. The consequences on microscopic expressions of the selfenergy are presented. The thermal averaged selfenergy and wave function renormalization, i.e. the frequency derivative of the selfenergy, are linked to the pair correlation function at small distances.

While all the expressions we derived are valid for finite temperatures, we have compared them as a test example with the Monte Carlo data for an electron gas at zero temperature. We find an improved description by the Pathak-Vashishta scheme accomplished by an energy shift derived from MC data. The best agreement with the data is achieved by constructing a nonlocal quasiparticle picture allowing for a wavevector-dependent effective mass and an energy shift in the form of a self-consistent quasiparticle picture.

\section{ACKNOWLEDGMENTS}

The fruitful discussions with Peter Fulde and Paul Ziesche are gratefully acknowledged. To Marco Ameduri I am indebted for a critical reading.

\section{APPENDIX A: DYNAMIC RESPONSE FUNCTION}

In the former paper 42 it was derived formally the density, current and energy response $\left\{\chi, \chi_{J}, \chi_{E}\right\}=$ $\mathcal{X}\{1,0,0\}^{T}$ of an interacting quantum system

$$
\left(\begin{array}{c}
\delta n \\
q \cdot \delta J \\
\delta E
\end{array}\right)=\left(\begin{array}{c}
\chi \\
\chi_{J} \\
\chi_{E}
\end{array}\right) V^{\mathrm{ext}} \equiv \mathcal{X}\left(\begin{array}{l}
1 \\
0 \\
0
\end{array}\right) V^{\mathrm{ext}}
$$

to the external perturbation $V^{\text {ext }}$ provided the density, momentum and energy are conserved. This has been achieved by linearizing the kinetic equation for the oneparticle density operator $\hat{\rho}$ in relaxation time approximation

$$
\dot{\hat{\rho}}+i\left[\hat{\mathcal{E}}+\hat{V}^{\mathrm{ext}}, \hat{\rho}\right]=\frac{\hat{\rho}^{\mathrm{l} . \mathrm{e}}-\hat{\rho}}{\tau}
$$

where the relaxation was considered with respect to the local density operator $\hat{\rho}^{\text {l.e. }}$ or the corresponding local equilibrium distribution function

$$
F(p)=\left[\left(\frac{\varepsilon_{0}(p-Q(R, t))-\mu(R, t)}{T(R, t)}\right)+1\right]^{-1} .
$$

This local equilibrium is given by a local time dependent chemical potential $\mu$, a local temperature $T$ and a local mass motion momentum $Q$. These local quantities have been specified by the requirement that the expectation values for density, momentum and energy are the same as the expectation values performed with $F$ which ensures conservation laws.

The correlations are shared in the kinetic equation (A2) in such a way that the energy operator $\hat{\mathcal{E}}$ parameterizes the density dependent quasiparticle energy or variation of the energy functional in the Landau liquid (momentum dependent) or density functional sense (momentum independent) and the collision integral is approximated by a conserving relaxation time approximation. While in 42 the general density, energy and momentum dependent form of such parameterization has been discussed we want to consider now only a special case of an effective mass and rigid shift parameterization

$$
\hat{\mathcal{E}}=-\nabla\left(\frac{1}{2 m(\hat{n})}\right) \nabla+\Delta(\hat{n})
$$

such that the variation $\mathcal{E}(n)=\mathcal{E}_{0}+\delta \mathcal{E}$ reads $\left[p=\left(p_{1}+\right.\right.$ $\left.\left.p_{2}\right) / 2, q=p_{1}-p_{2}\right]$

$$
\delta \mathcal{E}=<p_{1}|\delta \hat{\mathcal{E}}| p_{2}>=\left(V_{0}+V_{4} p^{2}\right) \delta n(q)
$$

with

$$
\begin{aligned}
& V_{0}=\frac{\delta}{\delta n} \Delta-V_{4} \frac{q^{2}}{4}+V_{q} \\
& V_{4}=\frac{\delta}{\delta n} \frac{1}{2 m}=-\frac{1}{2 n m} \frac{\partial \ln m}{\partial \ln n} .
\end{aligned}
$$

The response matrix (A1) can be given in terms of the polarization matrix $\mathcal{P}$, see Eq. (29) ot 42 , which is the response of the kinetic equation without the self-consistent quasiparticle energies $\delta \mathcal{E}$. The response reads

$$
\mathcal{X}=\left(\mathcal{I}-\mathcal{P} \mathcal{G}^{-1} \mathcal{V}\right)^{-1} \mathcal{P}
$$

with the matrices simplified for our considered case (A5)

$$
\begin{aligned}
& \mathcal{V}=\left(\begin{array}{ccc}
g_{1} V_{0}+g_{p^{2}} V_{4} & 0 & 0 \\
g_{\mathbf{p q}} V_{0}+g_{p^{2} \mathbf{p q}} V_{4} & 0 & 0 \\
g_{\epsilon} V_{0}++g_{p^{2} \epsilon} V_{4} & 0 & 0
\end{array}\right)_{\omega+\frac{i}{\tau}} \\
& \mathcal{G}=\left(\begin{array}{ccc}
g_{1} & g_{\mathbf{p q}} & g_{\epsilon} \\
g_{\mathbf{p q}} & g_{(\mathbf{p q})^{2}} & g_{\mathbf{p q} \epsilon} \\
g_{\epsilon} & g_{\epsilon \mathbf{p q}} & g_{\epsilon \epsilon}
\end{array}\right)_{\omega+\frac{i}{\tau}}
\end{aligned}
$$

and the correlation functions $\left[\varepsilon=p^{2} / 2 m+\Delta\right]$ are defined as

$$
g_{\phi}(\omega)=\int \frac{d p}{(2 \pi)^{3}} \phi \frac{F\left(p+\frac{q}{2}\right)-F\left(p-\frac{q}{2}\right)}{\varepsilon\left(p+\frac{q}{2}\right)-\varepsilon\left(p-\frac{q}{2}\right)-\omega-i 0} .
$$

Explicit formulae are in appendix B. We keep the matrix notation of 42 also for this special case in order to convince the reader about the technical usefulness of such notation. The $3 \times 3$ polarization matrix $\mathcal{P}=\left\{\Pi_{n m}\right\}$ contains the corresponding density, momentum and energy polarizations as

$$
\left(\begin{array}{c}
\delta n \\
\delta q \cdot J \\
\delta E
\end{array}\right)=\left(\begin{array}{c}
\Pi_{11} \\
\Pi_{21} \\
\Pi_{31}
\end{array}\right) V^{\text {ind }}
$$


In fact as found in 16 it is possible to express the density polarization function $\Pi_{11} \equiv \Pi^{\mathrm{n}, \mathrm{j}, \mathrm{E}}$ including density, current and energy conservation by a simpler one containing density and energy conservation $\Pi^{\mathrm{n}, \mathrm{E}}$ as

$$
\frac{1}{\Pi^{\mathrm{n}, \mathrm{j}, \mathrm{E}}(\omega)}-\frac{1}{\Pi^{\mathrm{n}, \mathrm{E}}(\omega)}=\frac{1}{\Pi^{\mathrm{n}, \mathrm{j}}(\omega)}-\frac{1}{\Pi^{\mathrm{n}}(\omega)}=-\frac{i \omega}{\tau} \frac{m}{n q^{2}} .
$$

This shows that the momentum conservation leads simply to a dynamical local field correction. The energy and density conserving polarization function reads explicitly

$$
\begin{aligned}
\Pi^{\mathrm{n}, \mathrm{E}}(\omega)= & (1-i \omega \tau)\left(\frac{g_{1}\left(\omega+\frac{i}{\tau}\right) g_{1}(0)}{h_{1}}\right. \\
& \left.-\omega \tau i \frac{\left(h_{\epsilon} g_{1}(0)-h_{1} g_{\epsilon}(0)\right)^{2}}{h_{1}\left(h_{\epsilon}^{2}-h_{\epsilon \epsilon} h_{1}\right)}\right)
\end{aligned}
$$

where we use the abbreviation $h_{\phi}=g_{\phi}\left(\omega+\frac{i}{\tau}\right)-$ $\omega \tau i g_{\phi}(0)$. The first part is just the known Mermin -Das pelinization function including only density conservation 5455

$$
\begin{aligned}
\Pi^{\mathrm{n}}(\omega) & =\frac{\Pi_{0}(\omega+i / \tau)}{1-\frac{1}{1-i \omega \tau}\left[1-\frac{\Pi_{0}(\omega+i / \tau)}{\Pi_{0}(0)}\right]} \\
& =(1-i \omega \tau) \frac{g_{1}\left(\omega+\frac{i}{\tau}\right) g_{1}(0)}{h_{1}}
\end{aligned}
$$

Now we want to give the full density response function $\chi=\mathcal{X}_{11}$ according to (A1). Due to the special considered case (A5) and consequently (A\&), the density response function $\chi$ can be written from (A7) into $\left[\Pi_{11}=\Pi^{\mathrm{n}, \mathrm{j}, \mathrm{E}}\right]$

$$
\chi(\omega)=\frac{\Pi^{\mathrm{n}, \mathrm{j}, \mathrm{E}}(\omega)}{1-V_{0} \Pi^{\mathrm{n}, \mathrm{j}, \mathrm{E}}(\omega)-2 m V_{4} \Pi_{13}(\omega)}
$$

where

$$
\begin{aligned}
\Pi_{13}(\omega) & =\frac{\Pi^{\mathrm{n}, \mathrm{j}, \mathrm{E}}}{2 m} \frac{\Pi_{h} \Pi_{2}(0)-i \tau \omega \Pi_{2}\left(\omega+\frac{i}{\tau}\right)}{\Pi_{h} \Pi_{0}(0)-i \tau \omega \Pi_{0}\left(\omega+\frac{i}{\tau}\right)}, \\
\Pi_{h} & =\frac{\Pi_{2}^{2}\left(\omega+\frac{i}{\tau}\right)-\Pi_{0}\left(\omega+\frac{i}{\tau}\right) \Pi_{4}\left(\omega+\frac{i}{\tau}\right)}{\Pi_{2}^{2}(0)-\Pi_{0}(0) \Pi_{4}(0)}
\end{aligned}
$$

are expressed in terms of moments of the correlation function (B1). The response function (33) is the main result of this paper since it gives the consistent response function for the quasiparticle consisting of effective mass, energy and relaxation time.

\section{APPENDIX B: EXPLICIT FORMULAE OF CORRELATION FUNCTIONS}

The different occurring correlation functions (A10) can be written in terms of moments of the usual Lindhard polarization function $\Pi_{0}$

$$
\Pi_{n}=s \int \frac{d \mathbf{p}}{(2 \pi)^{D}} p^{n} \frac{F\left(\mathbf{p}+\frac{\mathbf{q}}{2}\right)-F\left(\mathbf{p}-\frac{\mathbf{q}}{2}\right)}{\frac{\mathbf{p q}}{m}-\omega-i 0}
$$

as

$$
\begin{aligned}
g_{1} & =\Pi_{0} \\
g_{\mathbf{p q}} & =m \omega \Pi_{0} \\
g_{\epsilon} & =\frac{\Pi_{2}}{2 m} \\
g_{\epsilon p^{2}} & =\frac{\Pi_{4}}{2 m} \\
g_{p^{2} \mathbf{p q}} & =m \omega \Pi_{2} \\
g_{(\mathbf{p q})^{2}} & =-m q^{2} n+m^{2} \omega^{2} \Pi_{0} .
\end{aligned}
$$

Here $s$ is the spin degeneracy and $D$ gives the dimension of the system. While all formulae in the text are written for the three dimensional case they hold equally for one - and two dimensions.

For practical and numerical calculations we can rewrite the $\Pi_{n}$ by polynomial division into

$$
\begin{aligned}
\Pi_{2}= & -m n+\frac{m^{2} \omega^{2}}{q^{2}} \Pi_{0}+\tilde{\Pi}_{2} \\
\Pi_{4}= & -\frac{n m q^{2}}{4}\left(1+\frac{4 m^{2} \omega^{2}}{q^{4}}\right)-\frac{m^{4} \omega^{4}}{q^{4}} \Pi_{0} \\
& -\frac{2 m^{2} \omega^{2}}{q^{2}} \tilde{\Pi}_{2}-\tilde{\Pi}_{4}-\frac{14}{3} m^{2} E\left\{\begin{array}{cc}
1 & \text { for } D=2,3 \\
\frac{1}{2} & \text { for } D=1
\end{array}\right.
\end{aligned}
$$

where the $\tilde{\Pi}_{i}$ are the projected moments perpendicular to $\mathbf{q}$ and read

$$
\begin{aligned}
& \tilde{\Pi}_{2}=s \int \frac{d \mathbf{p}}{(2 \pi)^{D}}\left(\mathbf{p}-\frac{\mathbf{p q}}{q^{2}} \mathbf{q}\right)^{2} \frac{F\left(\mathbf{p}+\frac{\mathbf{q}}{2}\right)-F\left(\mathbf{p}-\frac{\mathbf{q}}{2}\right)}{\frac{\mathbf{p q}}{m}-\omega-i 0} \\
& =m \int_{-\infty}^{\mu} d \mu^{\prime} \Pi_{0} \times\left\{\begin{array}{cc}
2 & \text { for } D=3 \\
1 & \text { for } D=1,2
\end{array}\right\} \\
& \approx m T \Pi_{0} \times\left\{\begin{array}{cc}
2 & \text { for } D=3 \\
1 & \text { for } D=1,2
\end{array}\right\} \\
& \tilde{\Pi}_{4}=s \int \frac{d \mathbf{p}}{(2 \pi)^{D}}\left(\mathbf{p}-\frac{\mathbf{p q}}{q^{2}} \mathbf{q}\right)^{4} \frac{F\left(\mathbf{p}+\frac{\mathbf{q}}{2}\right)-F\left(\mathbf{p}-\frac{\mathbf{q}}{2}\right)}{\frac{\mathbf{p q}}{m}-\omega-i 0}
\end{aligned}
$$

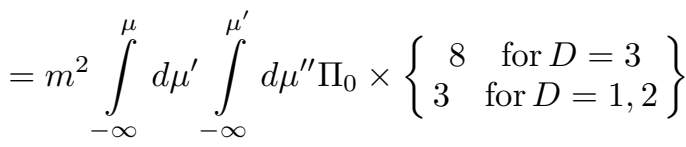

$$
\begin{aligned}
& \approx m^{2} T^{2} \Pi_{0} \times\left\{\begin{array}{cc}
8 & \text { for } D=3 \\
3 & \text { for } D=1,2
\end{array}\right\} .
\end{aligned}
$$

The corresponding last identities are valid only for nondegenerate, Maxwellian, distributions with temperature $T$. The general form of the polarization functions is presented as an integral over the chemical potential $\mu$ of the Lindhard polarization $\Pi_{0}$. This is applicable also to the degenerate case. 


\section{Long wavelength expansion}

In real situations it is often helpful to have the small wavevector expansion of the various occurring correlation functions. With the help of $(\sqrt{\mathrm{B} 4})$ and $(\mathrm{B} 3)$ this can be tremendously simplified if the expansion for $\Pi_{0}$ is written

$$
\begin{aligned}
\Pi_{0}(\omega)= & -\frac{s}{\omega} \int \frac{d p}{(2 \pi)^{D}}\left(-\frac{(q \cdot p)^{2}}{m^{2} \omega} F^{\prime}+\frac{q^{2}(q \cdot p)^{2}}{8 m^{3} \omega} F^{\prime \prime}\right. \\
& \left.-\frac{(q \cdot p)^{4}}{24 m^{4} \omega} F^{\prime \prime \prime}-\frac{(p \cdot q)^{4}}{m^{4} \omega^{3}} F^{\prime}\right)+o\left(q^{5}\right)
\end{aligned}
$$

where $F^{\prime}=\partial_{\mu} F$ etc. For the static case we have

$\Pi_{0}(0)=s \int \frac{d p}{(2 \pi)^{D}}\left(-F^{\prime}+\frac{q^{2}}{8 m} F^{\prime \prime}-\frac{(q \cdot p)^{2}}{24 m^{2}} F^{\prime \prime \prime}\right)+o\left(q^{4}\right)$.

We give now the one, two and three dimensional case separately.

\section{a. 3D case}

Since $\partial_{p} F=-p \partial_{\mu} F / m$ partial integration gives

$$
\int \frac{d p}{(2 \pi)^{3}} F^{\prime} G(p)=m \int \frac{d p}{(2 \pi)^{3}} \frac{F}{p^{2}} \partial_{p}(p G(p))
$$

and applied to (B5) one gets

$$
\Pi_{0}(\omega)=\frac{q^{2}}{m \omega^{2}} n+\frac{2 q^{4}}{m^{2} \omega^{4}} E+o\left(q^{6}\right) .
$$

The density, $n$, and energy, $E$, and higher moments read in terms of

$$
f_{n}=\frac{1}{\Gamma(n)} \int_{0}^{\infty} \frac{x^{n-1} d x}{\mathrm{e}^{x-\beta \mu}+1}
$$

as

$$
\begin{gathered}
n=\langle 1\rangle=\frac{s}{\lambda^{3}} f_{3 / 2} \\
E=\left\langle\frac{p^{2}}{2 m}\right\rangle=\frac{3}{2} \int^{\mu} d \mu^{\prime} n=\frac{3}{2 \beta} \frac{s}{\lambda^{3}} f_{5 / 2} \\
E_{2}=\left\langle\left(\frac{p^{2}}{2 m}\right)^{2}\right\rangle=\frac{5}{2} \int^{\mu} d \mu^{\prime} E=\frac{15}{4 \beta^{2}} \frac{s}{\lambda^{3}} f_{7 / 2} \\
E_{3}=\left\langle\left(\frac{p^{2}}{2 m}\right)^{3}\right\rangle=\frac{7}{2} \int^{\mu} d \mu^{\prime} E_{2}=\frac{105}{8 \beta^{3}} \frac{s}{\lambda^{3}} f_{9 / 2} .
\end{gathered}
$$

With the help of $(\sqrt{\mathrm{B} 4})$ and $(\mathrm{B} 3)$ one writes down immediately the higher order correlation functions as

$$
\begin{aligned}
& \tilde{\Pi}_{2}(\omega)=\frac{4 q^{2}}{3 \omega^{2}} E+\frac{8 q^{4}}{5 m \omega^{4}} E_{2} \\
& \tilde{\Pi}_{4}(\omega)=\frac{32 m q^{2}}{15 \omega^{2}} E_{2}+\frac{64 q^{4}}{35 \omega^{4}} E_{3} .
\end{aligned}
$$

The static case (B6) yields with B7

$$
\Pi_{0}(0)=-\partial_{\mu} n+\frac{q^{2}}{12 m} \partial_{\mu}^{2} n
$$

and with (B3) and (B4)

$$
\begin{aligned}
& \tilde{\Pi}_{2}(0)=-2 m n+\frac{q^{2}}{6} \partial_{\mu} n \\
& \tilde{\Pi}_{4}(0)=-\frac{16}{3} m^{2} E+\frac{2 m q^{2}}{3} n .
\end{aligned}
$$

During the text we use also the small and large wavevector limit of the static $3-\mathrm{D}$ polarization functions. Since

$$
\Pi_{0}(0)=\left\{\begin{array}{c}
-n^{2} \mathcal{K}_{0}+o\left(q^{2}\right) \\
-4 n \frac{m}{q^{2}}-\frac{32 m^{2}}{3 q^{4}} E+o\left(1 / q^{6}\right)
\end{array}\right.
$$

we obtain from (B3) and (B4)

$$
\Pi_{2}(0)=\left\{\begin{array}{c}
-3 m n+o\left(q^{2}\right) \\
-m n-\frac{16}{3} \frac{m^{2}}{q^{2}} E-\frac{128 m^{3}}{15 q^{4}} E_{2}+o\left(1 / q^{6}\right)
\end{array}\right.
$$

which leads to

$$
\frac{\Pi_{2}(0)}{\Pi_{0}(0)}=\left\{\begin{array}{c}
\frac{3 m}{n \mathcal{K}_{0}}+o\left(q^{2}\right) \\
\frac{q^{2}}{4}+\frac{2 E m}{3 n}+o\left(1 / q^{2}\right)
\end{array} .\right.
$$

\section{b. $2 D$ case}

For two dimensions we have instead of (B7)

$$
\int \frac{d p}{(2 \pi)^{2}} F^{\prime} G(p)=m \int \frac{d p}{(2 \pi)^{2}} F \partial_{p} G(p)
$$

which applied to (B5) yields

$$
\Pi_{0}(\omega)=\frac{2 \pi q^{2}}{m \omega^{2}} n+\frac{6 \pi q^{4}}{m^{2} \omega^{4}} E
$$

and from (B3) and (B4)

$$
\begin{aligned}
& \tilde{\Pi}_{2}(\omega)=\frac{2 \pi q^{2}}{\omega^{2}} E+\frac{6 \pi q^{4}}{m \omega^{4}} E_{2} \\
& \tilde{\Pi}_{4}(\omega)=\frac{6 \pi q^{2} m}{\omega^{2}} E_{2}+\frac{18 \pi q^{4}}{\omega^{4}} E_{3} .
\end{aligned}
$$

The different occurring moments reads here 


$$
\begin{gathered}
n=\langle 1\rangle=\frac{s}{\pi \lambda^{2}} f_{1} \\
E=\left\langle\frac{p^{2}}{2 m}\right\rangle=\int^{\mu} d \mu^{\prime} n=\frac{1}{\beta} \frac{s}{\lambda^{2}} f_{2} \\
E_{2}=\left\langle\left(\frac{p^{2}}{2 m}\right)^{2}\right\rangle=\int^{\mu} d \mu^{\prime} E=\frac{1}{\beta^{2}} \frac{s}{\lambda^{2}} f_{3} \\
E_{3}=\left\langle\left(\frac{p^{2}}{2 m}\right)^{3}\right\rangle=\int^{\mu} d \mu^{\prime} E_{2}=\frac{1}{\beta^{3}} \frac{s}{\lambda^{2}} f_{4} .
\end{gathered}
$$

The static case is now analogously and reads with (B17) from (B6)

$$
\begin{aligned}
& \Pi_{0}(0)=-\partial_{\mu} n+\frac{q^{2}}{24 m} \partial_{\mu}^{2} n \\
& \tilde{\Pi}_{2}(0)=-m n+\frac{q^{2}}{24} \partial_{\mu} n \\
& \tilde{\Pi}_{4}(0)=-3 m^{2} E+\frac{m q^{2}}{8} n .
\end{aligned}
$$

$$
\text { c. } 1 D \text { case }
$$

For one dimensions we have instead of (B7)

$$
\int \frac{d p}{(2 \pi)} F^{\prime} G(p)=m \int \frac{d p}{(2 \pi)} F \partial_{p}\left(\frac{G(p)}{p}\right)
$$

which applied to (B5) yields

$$
\begin{aligned}
& \Pi_{0}(\omega)=\frac{q^{2}}{3 m \omega^{2}} n-\frac{q^{4}}{60 m^{2} \omega^{2}} \partial_{\mu} n+\frac{6 q^{4}}{5 m^{2} \omega^{4}} E \\
& \tilde{\Pi}_{2}(\omega)=\frac{2 q^{2}}{3 \omega^{2}} E-\frac{q^{4}}{60 m \omega^{2}} n+\frac{4 q^{4}}{5 m \omega^{4}} E_{2} \\
& \tilde{\Pi}_{4}(\omega)=\frac{4 m q^{2}}{3 \omega^{2}} E_{2}-\frac{q^{4}}{10 \omega^{2}} E+\frac{24 q^{4}}{25 \omega^{4}} E_{3} .
\end{aligned}
$$

The static case is

$$
\begin{aligned}
& \Pi_{0}(0)=-\partial_{\mu} n+\frac{q^{2}}{12 m} \partial_{\mu}^{2} n \\
& \tilde{\Pi}_{2}(0)=-m n+\frac{q^{2}}{12} \partial_{\mu} n \\
& \tilde{\Pi}_{4}(0)=-=-6 m^{2} E+\frac{m q^{2}}{4} n .
\end{aligned}
$$

The occurring moments reads here

$$
\begin{gathered}
n=\langle 1\rangle=\frac{s}{4 \lambda} f_{1 / 2} \\
E=\left\langle\frac{p^{2}}{2 m}\right\rangle=\frac{1}{2} \int^{\mu} d \mu^{\prime} n=\frac{1}{8 \beta} \frac{s}{\lambda} f_{3 / 2} \\
E_{2}=\left\langle\left(\frac{p^{2}}{2 m}\right)^{2}\right\rangle=\frac{3}{2} \int^{\mu} d \mu^{\prime} E=\frac{3}{16 \beta^{2}} \frac{s}{\lambda} f_{5 / 2}
\end{gathered}
$$

$$
E_{3}=\left\langle\left(\frac{p^{2}}{2 m}\right)^{3}\right\rangle=\frac{5}{2} \int^{\mu} d \mu^{\prime} E_{2}=\frac{15}{32 \beta^{3}} \frac{s}{\lambda} f_{7 / 2} .
$$

\section{Large frequency limit}

The large frequency limit can be given analogously to the forgoing chapter. We restrict here to give the expansion for $3 \mathrm{D}$

$$
\Pi_{0}(\omega)=\frac{q^{2}}{m \omega^{2}} n+\frac{2 q^{4}}{m^{2} \omega^{4}} E+\frac{q^{6}}{4 m^{3} \omega^{4}} n+o\left(\frac{1}{\omega^{6}}\right) .
$$

Please remark the difference to the large wavelength expansion (B8). The corresponding higher order correlation functions are completely analogously given by the methods of the foregoing chapter. With (B4) and (B3) one gets

$$
\begin{aligned}
& \tilde{\Pi}_{2}(\omega)=\frac{4 q^{2}}{3 \omega^{2}} E+\frac{8 q^{4}}{5 m \omega^{4}} E_{2}+\frac{q^{6}}{3 m^{2} \omega^{4}} E+o\left(\frac{1}{\omega^{6}}\right) \\
& \tilde{\Pi}_{4}(\omega)=\frac{32 m q^{2}}{15 \omega^{2}} E_{2}+\frac{64 q^{4}}{35 \omega^{4}} E_{3}+\frac{8 q^{6}}{15 m \omega^{4}} E_{2}+o\left(\frac{1}{\omega^{6}}\right) .
\end{aligned}
$$

\section{APPENDIX C: PERTURBATION THEORY AND FREQUENCY SUM RULES FOR 1,2,3 DIMENSIONS}

The external potential is adiabatically switched on

$$
V^{\text {ext }}(r, t)=V(r) \mathrm{e}^{0 t} \Theta(-t)
$$

and induces a time dependent change in the Hamilton operator

$$
\delta \hat{H}(t)=-\int d r \hat{n}(r, t) V^{\mathrm{ext}}(r, t) .
$$

The variation of the density matrix operator $\hat{\rho}(t)=$ $\hat{\rho}+\delta \hat{\rho}(t)$ can be found from the linearized van-Neumann equation as

$$
\delta \hat{\rho}(t)=-i \int_{-\infty}^{t}\left[\delta \hat{H}, \hat{\rho}_{0}\right]
$$

where it has been assumed that the perturbation is conserving symmetries of the equilibrium Hamiltonian $\left[\hat{H}_{0}, \delta \hat{\rho}\right]=0$.

The variation of the density expectation value $\delta n=$ $\operatorname{Tr} \delta \rho \hat{n}$ is consequently 


$$
\delta n(r, t)=i \int_{-\infty}^{t} d t^{\prime} \int d r^{\prime} V\left(r^{\prime}, t^{\prime}\right)\left\langle\left[\hat{n}(r, t), \hat{n}\left(r^{\prime}, t^{\prime}\right)\right]\right\rangle
$$

Since in equilibrium the commutator is only dependent on the difference of coordinates and times we can define

$$
\begin{aligned}
-2 \operatorname{Im} \chi(q, \omega)= & \int d t \mathrm{e}^{i \omega\left(t-t^{\prime}\right)} \int d r d r^{\prime} \mathrm{e}^{-i q\left(r-r^{\prime}\right)} \\
& \times\left\langle\left[\hat{n}(r, t), \hat{n}\left(r^{\prime}, t^{\prime}\right)\right]\right\rangle
\end{aligned}
$$

from which we obtain the Fourier transform of (C4) to

$$
\delta n(q, \omega)=V^{\mathrm{ext}}(q, \omega) \int \frac{d \bar{\omega}}{\pi} \frac{\operatorname{Im} \chi(q, \bar{\omega})}{\bar{\omega}-\omega-i 0}
$$

where $V^{\text {ext }}(q, \omega)=V^{\text {ext }}(q) /(0+i \omega)$. This is of course identical with (11).

\section{Sum rules}

Now one can derive the first and second order sum rules of the imaginary part of the response function (C5). Therefore we generalize the definition (C5) to nonequilibrium and finite systems

$$
\begin{aligned}
& \operatorname{Im} \chi(q, \omega, R, t)=-\frac{1}{2} \int d \tau \mathrm{e}^{i \omega \tau} \int d r \mathrm{e}^{-i q r} \\
& \times\langle[\hat{n}(R+r / 2, t+\tau / 2), \hat{n}(R-r / 2, t-\tau / 2)]\rangle .
\end{aligned}
$$

Higher order moments can be expressed by correlation functions as well56. Here we restrict to the lowest two orders and rederive it in conventional way.

If we assume further equilibrium but finite systems we can define an averaged response by applying spatial averaging $\int d R / V$ to (C7) such that we obtain

$$
\operatorname{Im} \chi(q, \omega)=-\frac{1}{2 V} \int d \tau \mathrm{e}^{i \omega t}\langle[\hat{n}(q, t), \hat{n}(-q, 0)]\rangle .
$$

From this expression it is easy to see that the first two frequency sum rules read

$$
\begin{aligned}
& \int \frac{d \omega}{\pi} \omega \operatorname{Im} \chi(q, \omega)=-\frac{1}{V}\left\langle\left[\left.i \partial_{t} \hat{n}(q, t)\right|_{t=0}, \hat{n}(-q, 0)\right]\right\rangle \\
& \int \frac{d \omega}{\pi} \omega^{3} \operatorname{Im} \chi(q, \omega)=-\frac{1}{V}\left\langle\left[\left.\left(i \partial_{t}\right)^{3} \hat{n}(q, t)\right|_{t=0}, \hat{n}(-q, 0)\right]\right\rangle .
\end{aligned}
$$

Using the Heisenberg equation $i \partial_{t} \hat{n}=[\hat{n}, \hat{H}]$ and

$$
\begin{aligned}
\hat{H}= & \int \frac{d p}{(2 \pi)^{D}} \frac{p^{2}}{2 m} \hat{a}_{p}^{+} \hat{a}_{p} \\
& +\frac{1}{2} \int \frac{d p d p_{1} d p_{2}}{(2 \pi)^{3 D}} V_{p} \hat{a}_{p_{1}}^{+} \hat{a}_{p_{2}}^{+} \hat{a}_{p_{2}+p} \hat{a}_{p_{1}-p} \\
\hat{n}_{q}= & \int \frac{d p}{(2 \pi)^{D}} \hat{a}_{p}^{+} \hat{a}_{p+q}
\end{aligned}
$$

we can express the sum rules (C9) as

$$
\begin{aligned}
\int \frac{d \omega}{\pi} \omega \operatorname{Im} \chi(q, \omega) & =\frac{1}{V}\left\langle\left[q \hat{j}_{q}, \hat{n}_{-q}\right]\right\rangle \\
\int \frac{d \omega}{\pi} \omega^{3} \operatorname{Im} \chi(q, \omega) & =-\frac{1}{V}\left\langle\left[q \hat{j}_{q},\left[q \hat{j}_{-q}, \hat{H}\right]\right]\right\rangle
\end{aligned}
$$

where the divergence of the current operator reads

$$
q \hat{j}_{q}=\int \frac{d p}{(2 \pi)^{D}} \frac{2 p q+q^{2}}{2 m} \hat{a}_{p}^{+} \hat{a}_{p+q} .
$$

Performing the last commutators one obtains finally

$$
\int \frac{d \omega}{\pi} \omega \operatorname{Im} \chi(q, \omega)=\frac{q^{2}}{m} \frac{\left\langle\hat{n}_{q=0}\right\rangle}{V}=n \frac{q^{2}}{m}
$$

and

$$
\begin{aligned}
& \int \frac{d \omega}{\pi} \omega^{3} \operatorname{Im} \chi(q, \omega)=\frac{n q^{6}}{4 m^{3}}+\frac{3 q^{2}}{m^{3} V} \int \frac{d p}{(2 \pi)^{D}}(p \cdot q)^{2}\left\langle\hat{a}_{p}^{+} \hat{a}_{p}\right\rangle \\
& \frac{1}{m^{2} V} \int \frac{d p}{(2 \pi)^{D}} V_{p}\left\{(p \cdot q)^{2} \int \frac{d p_{1} d p_{2}}{(2 \pi)^{2 D}} \hat{a}_{p_{1}}^{+} \hat{a}_{p_{2}}^{+} \hat{a}_{p_{2}+p+q} \hat{a}_{p_{1}-p-q}\right. \\
& \left.-\left((p \cdot q)^{2}-q^{2}(p \cdot q)\right) \int \frac{d p_{1} d p_{2}}{(2 \pi)^{2 D}} \hat{a}_{p_{1}}^{+} \hat{a}_{p_{2}}^{+} \hat{a}_{p_{2}-p} \hat{a}_{p_{1}+p}\right\} .
\end{aligned}
$$

Since we had symmetric expressions $\phi(p)=\phi(-p)$ the second term leads just to kinetic energy density

$$
\begin{aligned}
& \frac{3 q^{2}}{m^{3} V} \int \frac{d p}{(2 \pi)^{D}}(p \cdot q)^{2}\left\langle\hat{a}_{p}^{+} \hat{a}_{p}\right\rangle \\
& =\frac{2 q^{4}}{m^{2} V} \int \frac{d p}{(2 \pi)^{D}} \frac{p^{2}}{2 m}\left\langle\hat{a}_{p}^{+} \hat{a}_{p}\right\rangle \times\left\{\begin{array}{cc}
3 & \text { for } 1 D \\
1 & \text { for } 2,3 D
\end{array}\right. \\
& =\frac{2 q^{2} E}{m^{2}} \times\left\{\begin{array}{cc}
3 & \text { for } 1 D \\
1 & \text { for } 2,3 D
\end{array} .\right.
\end{aligned}
$$

Now we are going to express the last 4 creation and annihilation operators by the structure function itself. Therefore we use the definition of the pair correlation function

$$
\left\langle\hat{a}_{r_{1}}^{+} \hat{a}_{r_{2}}^{+} \hat{a}_{r_{2}} \hat{a}_{r_{1}}\right\rangle=g_{r} n\left(r_{1}\right) n\left(r_{2}\right) .
$$

Applying the spatial averaging $\int d R / V$ where $R=\left(r_{1}+\right.$ $\left.r_{2}\right) / 2$ and Fourier-transform the difference $r_{1}-r_{2}$ into $q$ we obtain

$$
\begin{aligned}
& \int \frac{d p_{1} d p_{2}}{(2 \pi)^{2 D} V} \hat{a}_{p_{1}}^{+} \hat{a}_{p_{2}}^{+} \hat{a}_{p_{2}+q} \hat{a}_{p_{1}-q} \\
& =\int d r \mathrm{e}^{-i r \cdot q} g_{r} \int \frac{d R}{V} n(R+r / 2) n(R-r / 2) \\
& =n^{2} \int d r \mathrm{e}^{-i r \cdot q}\left(g_{r}-1\right)+n^{2}(2 \pi)^{3} \delta(q) \\
& =n\left(S_{q}-1\right)+n^{2}(2 \pi)^{3} \delta(q)
\end{aligned}
$$

where we neglected spatial gradients in the density and used (11) for the last step. Using (C17) in (C14) we obtain finally 


$$
\begin{aligned}
\int \frac{d \omega}{\pi} \omega^{3} \operatorname{Im} \chi(q, \omega) & =\frac{n q^{6}}{4 m^{3}}+\frac{2 q^{2} E}{m^{2}} \times\left\{\begin{array}{cc}
3 & \text { for } 1 D \\
1 & \text { for } 2,3 D
\end{array}\right\} \\
& -\frac{n^{2}}{m^{2}} q^{4} V_{q}\left(\tilde{I}(q)+\left\{\begin{array}{cc}
\tilde{I}_{1}(q) & \text { for } 1 D \\
0 & \text { for } 2,3 D
\end{array}\right\}\right)
\end{aligned}
$$

with (26)

$$
\tilde{I}(q)=-\frac{1}{n} \int \frac{d k}{(2 \pi)^{D}}\left(S_{k-q}-S_{k}+n \delta_{k, q}-n \delta_{k, 0}\right) \frac{(k \cdot q)^{2}}{q^{4}} \frac{V_{k}}{V_{q}}
$$

where we understand $\delta_{k}=(2 \pi)^{3} \delta(k)$. For the onedimensional case an extra term appears

$$
\tilde{I}_{1}(q)=\frac{1}{n} \int \frac{d k}{(2 \pi)}\left(S_{k}-1+n \delta_{k, 0}\right) \frac{k V_{k}}{q V_{q}} .
$$

In the following we restrict to the required formulae for the three dimensional case. One can Fourier transform

$$
\tilde{I}(q)=-\int \operatorname{drg}_{r}(1-\cos (q \cdot r)) \frac{\left(q \cdot \partial_{r}\right)^{2} V_{r}}{q^{4} V_{q}}
$$

which was first given by Puffl This correct form leads unavoidably to the appearance of the $\delta_{p}$ terms in (C19) very often overseen in later papers. For asymptotic expansions, however, we have to be careful that $g_{r}-1$ is the object which renders spatial integrals finite. Therefore the $\delta_{p}$ terms in (C19) have to be considered separately

$$
-\frac{1}{n} \int \frac{d k}{(2 \pi)^{3}}\left(n \delta_{k, q}-n \delta_{k, 0}\right) \frac{(k \cdot q)^{2}}{q^{4}} \frac{V_{k}}{V_{q}}=-1
$$

such that we obtain instead of C21

$$
\tilde{I}(q)=-\int d r\left(g_{r}-1\right)(1-\cos (q \cdot r)) \frac{\left(q \cdot \partial_{r}\right)^{2} V_{r}}{q^{4} V_{q}}-1 .
$$

For Coulomb potentials we can further simplify

$$
\tilde{I}(q)=-2 \int_{0}^{\infty} \frac{d r}{r}\left(g_{r}-1\right) j_{2}(q r)-1
$$

with the spherical Bessel function $j_{2}(x)$. From this expression one sees the small wavevector limit

$$
\begin{aligned}
\tilde{I}(q) & =-\frac{2 q^{2}}{15} \int_{0}^{\infty} d r r\left(g_{r}-1\right)-1+o\left(q^{4}\right) \\
& =\frac{2}{5} \frac{q^{2}}{k_{f}^{2}} \gamma-1+o\left(q^{4}\right)
\end{aligned}
$$

where we have used (11) and the definition (9). The long wavevector limit takes the form

$$
\begin{aligned}
\tilde{I}(q) & =-2 \int_{0}^{\infty} \frac{d x}{x}\left(g_{x / q}-1\right) j_{2}(x)-1 \\
& =-2\left(g_{0}-1\right) \int_{0}^{\infty} \frac{d x}{x} j_{2}(x)-1+o\left(1 / q^{2}\right) \\
& =\frac{2}{3}\left(1-g_{0}\right)-1+o\left(1 / q^{2}\right)
\end{aligned}
$$

${ }^{1}$ N. Iwamoto, E. Krotscheck, and D. Pines, Phys. Rev. B 29, 3936 (1984).

${ }^{2}$ L. V. Keldysh, D. A. Kirzhnitz, and A. A. Maradudin, The dielectric function of condensed systems (North-Holland, Amsterdam, 1989).

${ }^{3}$ J. Hubbard, Proc. R. Soc. A 243, 336 (1957).

${ }^{4}$ K. S. Singwi, M. P. Tosi, R. H. Land, and A. Sjölander, Phys. Rev. 176, 589 (1968).

${ }^{5}$ R. W. Shaw, Journ. Phys C 70, 1140 (1970).

${ }^{6}$ N. Studart, H. V. da Silveira, and U. deFreitas, Phys. Rev. E 53, 2350 (1996).

${ }^{7}$ K. N. Pathak and P. Vashishta, Phys. Rev. B 7, 3649 (1973).

${ }^{8}$ R. Puff, Phys. Rev. 137, A406 (1965).

${ }^{9}$ G. Niklasson, Phys. Rev. B 10, 3052 (1974).

${ }^{10}$ J. C. Kimball, Phys. Rev. A 7, 1648 (1973).

11 J. S. Vaishya and A. K. Gupta, Phys. Rev. B 7, 4300 (1973).

12 R. G. Dandrea, N. W. Ashcroft, and A. E. Carlsson, Phys. Rev. B 34, 2097 (1986).

${ }^{13}$ C. F. Richardson and N. W. Ashcroft, Phys. Rev. B 50, 8170 (1994).

${ }^{14}$ P. Vashishta and K. S. Singwi, Phys. Rev. B 6, 875 (1972).

${ }^{15}$ N. Iwamoto, Phys. Rev. A 30, 3289 (1984).

${ }^{16}$ H. K. Schweng and H. M. Böhm, Phys. Rev. B 48, 2037 (1993).

17 G. Röpke, R. Redmer, A. Wierling, and H. Reinholz, Phys. Rev. E 60, R2484 (1999).

${ }^{18}$ H. Reinholz, R. Redmer, G. Röpke, and A. Wierling, Phys. Rev. E 62, 5648 (2000).

${ }^{19}$ A. Holas and S. Rahman, Phys. Rev. B 35, 2720 (1987).

${ }^{20}$ B. Tanatar and C. Bulutay, Eur. Phys. J. B 12, 47 (1999).

${ }^{21}$ B. Tanatar and C. Bulutay, Phys. Rev. B 59, 15019 (1999).

22 C. Bowen, G. Sugiyama, and B. J. Alder, Phys. Rev. B 50, 14838 (1994).

${ }^{23}$ G. Ortiz and P. Ballone, Phys. Rev. B 50, 1391 (1994).

${ }^{24}$ S. Moroni, D. M. Ceperley, and G. Senatore, Phys. Rev. Lett. 75, 689 (1995).

${ }^{25}$ A. Gold, Z. Phys. B 103, 491 (1997).

${ }^{26}$ K. N. Pathak, S. Ranganathan, R. Bansal, and W. Bruns, Phys. Rev. A 31, 960 (1985).

${ }^{27}$ N. H. Kwong and M. Bonitz, Phys. Rev. Lett. 84, 1768 (2000).

${ }^{28}$ K. I. Golden and D. X. Lu, Phys. Rev. A 45, 1084 (1992). 
${ }^{29}$ L. Calmels and A. Gold, Phys. Rev. B 56, 1762 (1997).

30 J. G. Cordes and A. K. Das, Superlatt. and Microstr. 21, 195 (1997).

31 J. G. Cordes and A. K. Das, sol. state comm. 80, 145 (1991).

32 B. Tanatar and A. K. Das, Phys. Rev. B 54, 13827 (1996).

33 A. Nakano and S. Ichimaru, Phys. Rev. B 39, 4930 (1989).

${ }^{34}$ A. Nakano and S. Ichimaru, Phys. Rev. B 39, 4938 (1989).

${ }^{35}$ M. H. Lee, Phys. Rev. B 26, 2547 (1982).

${ }^{36}$ J. Hong and M. H. Lee, Phys. Rev. Lett. 70, 1972 (1993).

${ }^{37}$ K. Morawetz, R. Walke, and U. Fuhrmann, Phys. Rev. C 57, R 2813 (1998).

${ }^{38}$ K. Morawetz, U. Fuhrmann, and R. Walke, Nucl. Phys. A 649, 348 (1999).

39 S. Ichimaru, S. Mitake, S. Tanaka, and X. Z. Yan, Phys. Rev. A 32, 1768 (1985).

${ }^{40}$ G. Kalman and K. I. Golden, Phys. Rev. A 41, 5516 (1990).

${ }^{41}$ M. S. Morillo, Phys. of Plas. 7, 33 (2000).

${ }^{42}$ K. Morawetz and U. Fuhrmann, Phys. Rev. E 61, 2272 (2000).

${ }^{43}$ P. Ziesche, phys. stat. sol. (b) (2002) in press.

${ }^{44}$ P. Gori-Giorgi and P. Ziesche, (2002) cond-mat/0205342 submitted.

${ }^{45}$ D. Pines and P. Nozières, The Theory of Quantum Liquids (Addison-Wesley, New York, 1968), Vol. 1.

${ }^{46}$ K. Morawetz and U. Fuhrmann, Phys. Rev. E 62, 4382 (2000).

${ }^{47}$ E. K. U. Gross and W. Kohn, Phys. Rev. Lett. 55, 2850 (1985).

48 B. Dabrowski, Phys. Rev. B 34, 4989 (1986).

${ }^{49}$ B. Farid, V. Heine, G. E. Engel, and I. J. Robertson, Phys. Rev. B 48, 11602 (1993).

${ }^{50}$ R. Nifosi, S. Conti, and M. P. Tosi, Phys. Rev. B 58, 12758 (1998).

${ }^{51}$ S. Ichimaru, Rev. Mod. Phys. 54, 1017 (1982).

${ }^{52}$ M. Corradini, R. D. Sole, G. Onida, and M. Palummo, Phys. Rev. B 57, 14569 (1998).

${ }^{53}$ B. Davoudi, M. Polini, G. F. Giuliani, and M. P. Tosi, Phys. Rev. B 64, 153101 (2001).

${ }^{54}$ N. Mermin, Phys. Rev. B 1, 2362 (1970).

${ }^{55}$ A. K. Das, J. Phys. F 5, 2035 (1975).

${ }^{56}$ A. Manolescu and G. A. Mezincescu, Phys. Rev. A 37, 1760 (1988). 